\title{
Limit Cycle Bifurcations by Perturbing a Compound Loop with a Cusp and a Nilpotent Saddle
}

\author{
Huanhuan Tian and Maoan Han \\ Department of Mathematics, Shanghai Normal University, Shanghai 200234, China \\ Correspondence should be addressed to Maoan Han; mahan@shnu.edu.cn
}

Received 13 April 2014; Accepted 14 June 2014; Published 16 July 2014

Academic Editor: Tonghua Zhang

Copyright (C) 2014 H. Tian and M. Han. This is an open access article distributed under the Creative Commons Attribution License, which permits unrestricted use, distribution, and reproduction in any medium, provided the original work is properly cited.

\begin{abstract}
We study the expansions of the first order Melnikov functions for general near-Hamiltonian systems near a compound loop with a cusp and a nilpotent saddle. We also obtain formulas for the first coefficients appearing in the expansions and then establish a bifurcation theorem on the number of limit cycles. As an application example, we give a lower bound of the maximal number of limit cycles for a polynomial system of Liénard type.
\end{abstract}

\section{Introduction}

Consider a planar system of the form

$$
\dot{x}=H_{y}+\epsilon p(x, y, \delta), \quad \dot{y}=-H_{x}+\epsilon q(x, y, \delta),
$$

where $\epsilon$ is a small parameter and $H(x, y), p(x, y, \delta)$, and $q(x, y, \delta)$ are $C^{\infty}$ functions in $(x, y) \in \mathbb{R}^{2}$ and $\delta \in D \subset \mathbb{R}^{m}$ with $D$ bounded. For $\epsilon=0$, (1) becomes

$$
\dot{x}=H_{y}, \quad \dot{y}=-H_{x},
$$

which is a Hamiltonian system. As we know, the system (1) is said to be a near-Hamiltonian system. For (1), the main task is to study the number of limit cycles which are bifurcated from periodic orbits of the unperturbed system (2). On this aspect, the first order Melnikov function of (1) plays an important role. We can use the expansions of it near Hamiltonian values corresponding to a center or an invariant loop to find its zeros and hence the number of limit cycles. See a survey article [1]. There have been many works on this topic. For the study of general near-Hamiltonian systems, see [2-12]; and especially for the system (2) with the elliptic case, one can see [13-17] and references therein. In [2-4], the number of limit cycles of the system (1) near a homoclinic loop with a cusp of order one or two or a nilpotent saddle of order one (for the definition of an order of a cusp or nilpotent saddle, see [5]) was studied. In the heteroclinic case with two hyperbolic saddles, a hyperbolic saddle and a cusp of order one, or two cusps of order one or two, the number of limit cycles of the system (1) was studied in $[5,8,9]$, respectively. In this paper, we suppose that the unperturbed system (2) has a compound loop consisting of a cusp $S_{1}$ of order one, a nilpotent saddle $S_{2}$ of order one, a homoclinic loop to $S_{2}$, and two heteroclinic orbits connecting $S_{1}$ and $S_{2}$, as shown in Figure 1. We aim to study the number of limit cycles of (1) near the loop for $\epsilon \neq 0$ small.

\section{Main Results with Proof}

Now consider the $C^{\infty}$ systems (1) and (2). Suppose that (2) has a compound loop denoted by $L_{0}=L_{1} \cup L_{2} \cup L_{3} \cup\left\{S_{1}, S_{2}\right\}$ and defined by equation $H(x, y)=0$, where $S_{1}\left(x_{1}, y_{1}\right)$ is a cusp and $S_{2}\left(x_{2}, y_{2}\right)$ is a nilpotent saddle both having order one, $L_{1}, L_{2}$ are heteroclinic orbits satisfying $\omega\left(L_{1}\right)=\alpha\left(L_{2}\right)=$ $S_{2}$ and $\omega\left(L_{2}\right)=\alpha\left(L_{1}\right)=S_{1}$, and $L_{3}$ is a homoclinic loop to $S_{2}$. Then, the level curves of $H(x, y)$ define two families of periodic orbits $L_{h 1}$ and $L_{h 2}$ for $h$ on one side of $h=0$ and a family of periodic orbits $L_{h 3}$ for $h$ on another side of $h=0$. For the definiteness, let both $L_{h 1}$ and $L_{h 2}$ exist for $0<-h \ll 1$ and $L_{h 3}$ exist for $0<h \ll 1$. Thus, we have three Melnikov functions

$$
M_{i}(h, \delta)=\oint_{L_{h i}} q d x-p d y, \quad i=1,2,3
$$




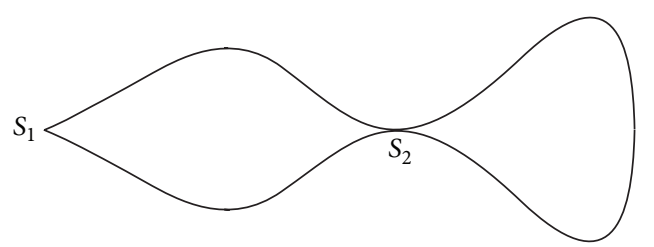

FIgURE 1: Compound loop with a cusp and a nilpotent saddle.

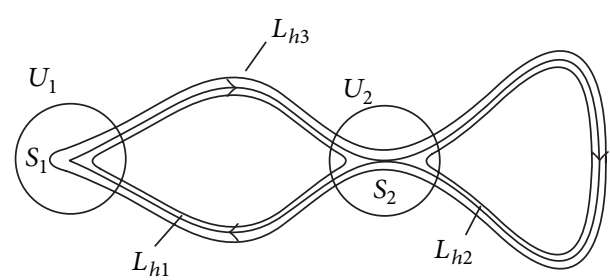

(a)

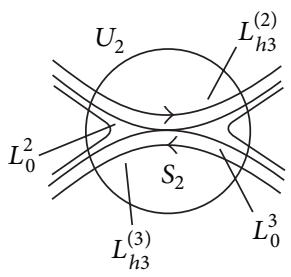

(b)

Figure 2

Let $U_{i}$ denote a closed set with diameter $\epsilon_{0}>0$ and with center at $S_{i}, i=1,2$. See Figure 2(a). And further introduce

$$
\begin{gathered}
L_{h 1}^{(j)}=L_{h 1} \cap U_{j}, \quad j=1,2, \\
L_{h 1}^{(3)}=\mathrm{Cl} .\left(L_{h 1}-\bigcup_{j=1}^{2} L_{h 1}^{(j)}\right), \quad L_{h 2}^{(1)}=L_{h 2} \cap U_{2}, \\
L_{h 2}^{(2)}=L_{h 2}-L_{h 2}^{(1)}, \quad L_{h 3}^{(1)}=L_{h 3} \cap U_{1}, \\
L_{h 3} \cap U_{2}=L_{h 3}^{(2)} \cup L_{h 3}^{(3)} \quad(\text { as shown in Figure } 2(\mathrm{~b})), \\
L_{h 3}^{(4)}=\mathrm{Cl} .\left(L_{h 3}-\bigcup_{j=1}^{3} L_{h 3}^{(j)}\right) .
\end{gathered}
$$

Here the $\mathrm{Cl}$. denotes the closure of a set. Then by (3) and (4), for $\epsilon_{0}$ sufficiently small we can write

$$
\begin{aligned}
& M_{1}(h, \delta)=I_{11}(h, \delta)+I_{12}(h, \delta)+I_{13}(h, \delta) \\
& \qquad \text { for } 0 \leq-h \ll 1, \\
& M_{2}(h, \delta)=I_{21}(h, \delta)+I_{22}(h, \delta) \\
& \text { for } 0 \leq-h \ll 1, \\
& M_{3}(h, \delta)=I_{31}(h, \delta)+I_{32}(h, \delta)+I_{33}(h, \delta)+I_{34}(h, \delta) \\
& \text { for } 0 \leq h \ll 1,
\end{aligned}
$$

where

$$
I_{i j}(h, \delta)=\int_{L_{h i}^{(j)}} q d x-p d y, \quad i \in\{1,2,3\}, j \in\{1,2,3,4\} .
$$

By [5], there exist two transformations of the form

$$
(x, y)^{T}=T_{i}(u, v)^{T}+S_{i}, \quad i=1,2,
$$

where $T_{i}$ is a $2 \times 2$ matrix satisfying $\operatorname{det} T_{i}=1$ such that (1) becomes

$$
\dot{u}=H_{i v}+\epsilon p_{i}(u, v, \delta), \quad \dot{v}=-H_{i u}+\epsilon q_{i}(u, v, \delta),
$$

where

$$
\begin{gathered}
H_{1}(u, v)=\frac{v^{2}}{2}+\sum_{k+j \geq 3} \tilde{h}_{k j} u^{k} v^{j}, \\
H_{1}\left(u, \varphi_{1}(u)\right)=\widetilde{h}_{3} u^{3}+O\left(u^{4}\right), \quad \tilde{h}_{3}<0, \\
H_{1 v}\left(u, \varphi_{1}(u)\right)=0, \quad p_{1}(u, v, \delta)=\sum_{i+j \geq 0} \tilde{a}_{i j} u^{i} v^{j}, \\
q_{1}(u, v, \delta)=\sum_{i+j \geq 0} \tilde{b}_{i j} u^{i} v^{j}, \\
H_{2}(u, v)=\frac{v^{2}}{2}+\sum_{k+j \geq 3} \bar{h}_{k j} u^{k} v^{j}, \\
H_{2}\left(u, \varphi_{2}(u)\right)=\bar{h}_{4} u^{4}+O\left(u^{5}\right), \quad \bar{h}_{4}<0, \\
H_{2 v}\left(u, \varphi_{2}(u)\right)=0, \\
p_{2}(u, v, \delta)=\sum_{i+j \geq 0} \bar{a}_{i j} u^{i} v^{j}, \\
q_{2}(u, v, \delta)=\sum_{i+j \geq 0} \bar{b}_{i j} u^{i} v^{j},
\end{gathered}
$$


for $(u, v)$ near $(0,0)$. Note that $q d x-p d y=q_{1} d u-p_{1} d v$ for $(x, y)$ near $S_{1}$ and $q d x-p d y=q_{2} d u-p_{2} d v$ for $(x, y)$ near $S_{2}$. Then we have

$$
\begin{aligned}
& I_{i 1}(h, \delta)=\int_{\tilde{L}_{h i}^{(1)}} q_{1} d u-p_{1} d v, \quad i=1,3, \\
& I_{21}(h, \delta)=\int_{\bar{L}_{h 2}^{(1)}} q_{2} d u-p_{2} d v, \\
& I_{12}(h, \delta)=\int_{\bar{L}_{h 1}^{(2)}} q_{2} d u-p_{2} d v, \\
& I_{3 j}(h, \delta)=\int_{\bar{L}_{h 3}^{(j)}} q_{2} d u-p_{2} d v, \quad j=2,3,
\end{aligned}
$$

where $\widetilde{L}_{h i}^{(1)}$ denote the image of $L_{h i}^{(1)}$ under $T_{1}$ and $\bar{L}_{h 2}^{(1)}, \bar{L}_{h 1}^{(2)}$, and $\bar{L}_{h 3}^{(j)}$ denote the image of $L_{h 2}^{(1)}, L_{h 1}^{(2)}$, and $L_{h 3}^{(j)}$ under $T_{2}$, respectively. Then, by using $[3,4]$ we can obtain the following two lemmas, respectively.

Lemma 1. Consider system (10) with $i=1$ and suppose (11), (13) hold. Then there are constants $B_{00}, B_{00}^{*}, B_{10}, B_{10}^{*}$ satisfying

$$
\begin{gathered}
B_{00}=\frac{3}{5} \int_{0}^{1} \frac{d v}{\sqrt{v\left(1-v^{3}\right)}}=\frac{3}{5} \times 2.4286 \cdots>0, \\
B_{00}^{*}=-\frac{3}{5} \int_{-\infty}^{1} \frac{d v}{\sqrt{1-v^{3}}}=-\frac{3}{5} \times 4.2065 \cdots<0, \\
B_{10}=-\frac{3}{7}\left(\int_{0}^{1} \frac{v^{3 / 2} d v}{\sqrt{1-v^{3}}\left(1+\sqrt{1-v^{3}}\right)}-2\right)>0, \\
B_{10}^{*} \\
=\frac{3}{7}\left(\int_{1}^{-1} \frac{v d v}{\sqrt{1-v^{3}}}-\int_{0}^{1} \frac{v^{3 / 2} d v}{\sqrt{1+v^{3}}\left(1+\sqrt{1+v^{3}}\right)}-2\right)<0
\end{gathered}
$$

such that

$$
\begin{aligned}
I_{11}(h, \delta)= & B_{00} c_{1}\left(S_{1}, \delta\right)|h|^{5 / 6}+B_{10} c_{3}\left(S_{1}, \delta\right)|h|^{7 / 6} \\
& -\frac{1}{11} B_{00} c_{4}\left(S_{1}, \delta\right)|h|^{11 / 6}+O\left(h^{2}\right)+N_{11}(h, \delta)
\end{aligned}
$$

for $0<-h \ll 1$,

$$
\begin{aligned}
I_{31}(h, \delta)= & B_{00}^{*} c_{1}\left(S_{1}, \delta\right) h^{5 / 6}+B_{10}^{*} c_{3}\left(S_{1}, \delta\right) h^{7 / 6} \\
& +\frac{1}{11} B_{00}^{*} c_{4}\left(S_{1}, \delta\right) h^{11 / 6}+O\left(h^{2}\right)+N_{31}(h, \delta)
\end{aligned}
$$

for $0<h \ll 1$, where $N_{i 1}(h, \delta) \in C^{\omega}$ at $h=0$ with $N_{i 1}(0, \delta)=$ $O\left(\epsilon_{0}\right), i=1,3$, and

$$
\begin{aligned}
c_{1}\left(S_{1}, \delta\right)=2 & \sqrt{2} \widetilde{h}_{3}^{-1 / 3}\left(\widetilde{a}_{10}+\widetilde{b}_{01}\right), \\
c_{3}\left(S_{1}, \delta\right)=2 \sqrt{2} \widetilde{h}_{3}^{-5 / 3} & \\
& \times\left[\widetilde{h}_{3}\left(2 \widetilde{a}_{20}+\widetilde{b}_{11}\right)\right. \\
& \left.+\frac{1}{3}\left(\widetilde{h}_{21}^{2}-2 \widetilde{h}_{4}-3 \widetilde{h}_{3} \widetilde{h}_{12}\right)\left(\widetilde{a}_{10}+\widetilde{b}_{01}\right)\right], \\
c_{4}\left(S_{1}, \delta\right)= & 9 \mu_{1}^{-1} \widetilde{\alpha}_{01} \\
& -2 \mu_{1}^{-7}\left[\left(20 \mu_{2}^{3}-20 \mu_{1} \mu_{2} \mu_{3}+4 \mu_{1}^{2} \mu_{4}\right) \widetilde{\alpha}_{00}\right. \\
& +\left(4 \mu_{1}^{2} \mu_{3}-10 \mu_{1} \mu_{2}^{2}\right) \widetilde{\alpha}_{10} \\
& \left.+4 \mu_{1}^{2} \mu_{2} \widetilde{\alpha}_{20}-\mu_{1}^{3} \widetilde{\alpha}_{30}\right],
\end{aligned}
$$

where

$$
\begin{gathered}
\mu_{1}=\widetilde{h}_{3}^{1 / 3}, \quad \mu_{2}=\frac{1}{3} \widetilde{h}_{3}^{-2 / 3} \widetilde{h}_{4}, \\
\mu_{3}=\frac{1}{9} \widetilde{h}_{3}^{-5 / 3}\left(3 \widetilde{h}_{3} \widetilde{h}_{5}-\widetilde{h}_{4}^{2}\right), \\
\mu_{4}=\frac{1}{81} \widetilde{h}_{3}^{-8 / 3}\left(27 \widetilde{h}_{3}^{2} \widetilde{h}_{6}-18 \widetilde{h}_{3} \widetilde{h}_{4} \widetilde{h}_{5}+5 \widetilde{h}_{4}^{3}\right), \\
\widetilde{h}_{3}=\widetilde{h}_{30}, \quad \widetilde{h}_{4}=-\frac{1}{2} \widetilde{h}_{21}^{2}+\widetilde{h}_{40}, \\
\widetilde{h}_{5}=\widetilde{h}_{12} \widetilde{h}_{21}^{2}-\widetilde{h}_{21} \widetilde{h}_{31}+\widetilde{h}_{50}, \\
\widetilde{h}_{6}=-2 \widetilde{h}_{12}^{2} \widetilde{h}_{21}^{2}-\widetilde{h}_{03} \widetilde{h}_{21}^{3}+\widetilde{h}_{21}^{2} \widetilde{h}_{22} \\
+2 \widetilde{h}_{12} \widetilde{h}_{21} \widetilde{h}_{31}-\frac{1}{2} \widetilde{h}_{31}^{2}-\widetilde{h}_{21} \widetilde{h}_{41}+\widetilde{h}_{60}, \\
\widetilde{\alpha}_{00}=2 \sqrt{2}\left(\widetilde{a}_{10}+\widetilde{b}_{01}\right), \\
\widetilde{\alpha}_{10}=2 \sqrt{2}\left[-\widetilde{h}_{12}\left(\widetilde{a}_{10}+\widetilde{b}_{01}\right)+2 \widetilde{a}_{20}+\widetilde{b}_{11}\right],
\end{gathered}
$$

$\widetilde{\alpha}_{20}$

$$
\begin{gathered}
=2 \sqrt{2}\left[\left(\widetilde{a}_{10}+\widetilde{b}_{01}\right)\left(3 \widetilde{h}_{03} \widetilde{h}_{21}-\widetilde{h}_{22}+\frac{3}{2} \widetilde{h}_{12}^{2}\right)-2 \widetilde{h}_{12} \widetilde{a}_{20}\right. \\
\left.-\widetilde{h}_{12} \widetilde{b}_{11}+3 \widetilde{a}_{30}+\widetilde{b}_{21}-\widetilde{a}_{11} \widetilde{h}_{21}-2 \widetilde{b}_{02} \widetilde{h}_{21}\right],
\end{gathered}
$$

$\widetilde{\alpha}_{30}$

$$
\begin{gathered}
=2 \sqrt{2}\left[( \widetilde { a } _ { 1 0 } + \widetilde { b } _ { 0 1 } ) \left(3 \widetilde{h}_{13} \widetilde{h}_{21}+3 \widetilde{h}_{03} \widetilde{h}_{31}+3 \widetilde{h}_{12} \widetilde{h}_{22}\right.\right. \\
\left.-15 \widetilde{h}_{12} \widetilde{h}_{03} \widetilde{h}_{21}-\frac{5}{2} \widetilde{h}_{12}^{3}-\widetilde{h}_{32}\right) \\
+\left(3 \widetilde{a}_{11}+6 \widetilde{b}_{02}\right) \widetilde{h}_{12} \widetilde{h}_{21}-2\left(\widetilde{b}_{12}+\widetilde{a}_{21}\right) \widetilde{h}_{21} \\
-\left(\widetilde{a}_{11}+2 \widetilde{b}_{02}\right) \widetilde{h}_{31}+4 \widetilde{a}_{40}+\widetilde{b}_{31}
\end{gathered}
$$




$$
\begin{array}{r}
+\left(3 \widetilde{b}_{11}+6 \widetilde{a}_{20}\right) \tilde{h}_{03} \tilde{h}_{21}-\left(2 \widetilde{a}_{20}+\widetilde{b}_{11}\right) \widetilde{h}_{22} \\
\left.+\left(3 \widetilde{a}_{20}+\frac{3}{2} \widetilde{b}_{11}\right) \tilde{h}_{12}^{2}-\left(3 \widetilde{a}_{30}+\widetilde{b}_{21}\right) \widetilde{h}_{12}\right] \\
\widetilde{\alpha}_{01}=2 \sqrt{2}\left[\frac{2}{3} \widetilde{a}_{12}+2 \widetilde{b}_{03}-2 \widetilde{h}_{03} \widetilde{a}_{11}\right. \\
\left.\quad-4 \widetilde{h}_{03} \widetilde{b}_{02}+\left(\widetilde{a}_{10}+\widetilde{b}_{01}\right)\left(5 \widetilde{h}_{03}^{2}-2 \widetilde{h}_{04}\right)\right] .
\end{array}
$$

Lemma 2. Consider system (10) with $i=2$ and suppose (12), (15) hold. Then we have

$$
\begin{aligned}
I_{12}(h, \delta)= & c_{1}\left(S_{2}, \delta\right)|h|^{3 / 4}-c_{2}\left(S_{2}, \delta\right) h \ln |h| \\
& +c_{4}\left(S_{2}, \delta\right)|h|^{5 / 4}+c_{5}\left(S_{2}, \delta\right)|h|^{7 / 4} \\
& -c_{6}\left(S_{2}, \delta\right) h^{2} \ln |h|+O\left(h^{2}\right)+N_{12}(h, \delta)
\end{aligned}
$$

for $0<-h \ll 1$,

$$
\begin{aligned}
I_{32}(h, \delta)= & \frac{1}{2} c_{1}^{*}\left(S_{2}, \delta\right) h^{3 / 4}+\frac{1}{2} c_{3}^{*}\left(S_{2}, \delta\right) h^{5 / 4} \\
& +\frac{1}{2} c_{4}^{*}\left(S_{2}, \delta\right) h^{7 / 4}+O\left(h^{2}\right)+N_{32}(h, \delta), \\
I_{33}(h, \delta)= & \frac{1}{2} c_{1}^{*}\left(S_{2}, \delta\right) h^{3 / 4}+\frac{1}{2} c_{3}^{*}\left(S_{2}, \delta\right) h^{5 / 4} \\
& +\frac{1}{2} c_{4}^{*}\left(S_{2}, \delta\right) h^{7 / 4}+O\left(h^{2}\right)+N_{33}(h, \delta)
\end{aligned}
$$

for $0<h \ll 1$, where $N_{i j}(h, \delta) \in C^{\omega}$ at $h=0$ with $N_{i j}(0, \delta)=$ $O\left(\epsilon_{0}\right),(i, j) \in\{(1,2),(3,2),(3,3)\}$, and

$$
\begin{gathered}
c_{1}\left(S_{2}, \delta\right)=-2 \sqrt{2}\left|\widetilde{A}_{0}\right|\left|\bar{h}_{4}\right|^{-1 / 4}\left(\bar{a}_{10}+\bar{b}_{01}\right) \\
c_{2}\left(S_{2}, \delta\right)=-\frac{\sqrt{2}}{4}\left|\bar{h}_{4}\right|^{-1 / 2}\left(2 \bar{a}_{20}+\bar{b}_{11}\right)+O\left(\bar{a}_{10}+\bar{b}_{01}\right), \\
c_{4}\left(S_{2}, \delta\right)=\left|\widetilde{A}_{2}\right|\left[\left(\frac{21}{32} \bar{h}_{5}^{2}-\frac{3}{4} \bar{h}_{4} \bar{h}_{6}\right)\left|\bar{h}_{4}\right|^{-11 / 4} \bar{\alpha}_{00}\right. \\
\left.+\frac{3}{4}\left|\bar{h}_{4}\right|^{-7 / 4} \bar{h}_{5} \bar{\alpha}_{10}+\left|\bar{h}_{4}\right|^{-3 / 4} \bar{\alpha}_{20}\right], \\
c_{5}\left(S_{2}, \delta\right)=\frac{1}{7}\left|\widetilde{A}_{0}\right|\left[6 d_{1}^{-1} \bar{\alpha}_{01}\right. \\
+d_{1}^{-9}\left(105 d_{1} d_{2}^{2} d_{3}-30 d_{1}^{2} d_{2} d_{4}\right. \\
\left.-15 d_{1}^{2} d_{3}^{2}+5 d_{1}^{3} d_{5}-70 d_{2}^{4}\right) \bar{\alpha}_{00} \\
+d_{1}^{-8}\left(35 d_{2}^{3}-30 d_{1} d_{2} d_{3}+5 d_{1}^{2} d_{4}\right) \bar{\alpha}_{10} \\
-d_{1}^{-7}\left(15 d_{2}^{2}-5 d_{1} d_{3}\right) \bar{\alpha}_{20} \\
\left.+5 d_{1}^{-6} d_{2} \bar{\alpha}_{30}-d_{1}^{-5} \bar{\alpha}_{40}\right],
\end{gathered}
$$

$$
\begin{gathered}
c_{6}\left(S_{2}, \delta\right) \quad-\frac{1}{32}\left[-6 d_{1}^{-3} d_{2} \bar{\alpha}_{01}+3 d_{1}^{-2} \bar{\alpha}_{11}\right. \\
-d_{1}^{-11}\left(504 d_{1} d_{2}^{3} d_{3}-168 d_{1}^{2} d_{2} d_{3}^{2}\right. \\
+42 d_{1}^{3} d_{3} d_{4}-168 d_{1}^{2} d_{2}^{2} d_{4} \\
\left.+42 d_{1}^{3} d_{2} d_{5}-252 d_{2}^{5}-6 d_{1}^{4} d_{6}\right) \bar{\alpha}_{00} \\
+d_{1}^{-10}\left(168 d_{1} d_{2}^{2} d_{3}-126 d_{2}^{4}\right. \\
\left.-42 d_{1}^{2} d_{2} d_{4}-21 d_{1}^{2} d_{3}^{2}+6 d_{1}^{3} d_{5}\right) \bar{\alpha}_{10} \\
-d_{1}^{-9}\left(42 d_{1} d_{2} d_{3}-56 d_{2}^{3}-6 d_{1}^{2} d_{4}\right) \bar{\alpha}_{20} \\
+d_{1}^{-8}\left(6 d_{1} d_{3}-21 d_{2}^{2}\right) \bar{\alpha}_{30} \\
\left.+6 d_{1}^{-7} d_{2} \bar{\alpha}_{40}-d_{1}^{-6} \bar{\alpha}_{50}\right] \\
c_{1}^{*}\left(S_{2}, \delta\right)=-D_{1} c_{1}\left(S_{2}, \delta\right), \\
c_{4}^{*}\left(S_{2}, \delta\right)=D_{1} c_{5}\left(S_{2}, \delta\right)
\end{gathered}
$$

where $D_{1}=2\left|\bar{A}_{0}\right| /\left|\widetilde{A}_{0}\right|, D_{2}=2\left|\bar{A}_{1}\right| /\left|\widetilde{A}_{2}\right|, \bar{A}_{0}, \widetilde{A}_{0}, \bar{A}_{1}$, and $\widetilde{A}_{2}$ are constants, given by

$$
\begin{aligned}
\bar{A}_{0} & =\frac{2}{3} \int_{0}^{\infty} \frac{d v}{\sqrt{1+v^{4}}} \approx 1.236049785>0, \\
\widetilde{A}_{0} & =-\frac{2}{3} \int_{0}^{1} \frac{d v}{\sqrt{1-v^{4}}} \\
& =-\frac{\sqrt{2} \pi^{3 / 2}}{6[\Gamma(3 / 4)]^{2}} \approx-0.8740191847<0, \\
\bar{A}_{1} & =-\frac{2}{5} \int_{0}^{\infty} \frac{d v}{\sqrt{1+v^{4}}\left[v^{2}+\sqrt{1+v^{4}}\right]} \\
& \approx-0.3388852337<0, \\
\widetilde{A}_{2} & =\frac{2}{5}\left[1-\int_{0}^{1} \frac{v^{2} d v}{\sqrt{1-v^{4}}\left(1+\sqrt{1-v^{4}}\right)}\right] \\
& \approx 0.2396280470>0, \\
d_{4}=- & \frac{1}{128}\left|\bar{h}_{4}\right|^{-11 / 4}\left(7 \bar{h}_{5}^{3}+32 \bar{h}_{4}^{2} \bar{h}_{7}-24 \bar{h}_{4} \bar{h}_{5} \bar{h}_{6}\right), \\
& d_{3}=\frac{1}{32}\left|\bar{h}_{4}\right|^{-7 / 4}\left(8 \bar{h}_{4} \bar{h}_{6}-3 \bar{h}_{5}^{2}\right),-\frac{1}{4}\left|\bar{h}_{4}\right|^{-3 / 4} \bar{h}_{5}, \\
d_{1} &
\end{aligned}
$$




$$
\begin{aligned}
& d_{5}=\frac{1}{2048}\left|\bar{h}_{4}\right|^{-15 / 4}\left(512 \bar{h}_{4}^{3} \bar{h}_{8}-192 \bar{h}_{4}^{2} \bar{h}_{6}^{2}\right. \\
& \bar{\alpha}_{50}=2 \sqrt{2}\left[n_{2}\left(\bar{a}_{10}+\bar{b}_{01}\right)+n_{1}\left(2 \bar{a}_{20}+\bar{b}_{11}\right)\right. \\
& \left.-384 \bar{h}_{4}^{2} \bar{h}_{5} \bar{h}_{7}+336 \bar{h}_{4} \bar{h}_{5}^{2} \bar{h}_{6}-77 \bar{h}_{5}^{4}\right), \\
& d_{6}=-\frac{1}{8192}\left|\bar{h}_{4}\right|^{-19 / 4}\left(2048 \bar{h}_{4}^{4} \bar{h}_{9}+1344 \bar{h}_{4}^{2} \bar{h}_{5} \bar{h}_{6}^{2}\right. \\
& -1536 \bar{h}_{4}^{3} \bar{h}_{5} \bar{h}_{8}-1536 \bar{h}_{4}^{3} \bar{h}_{6} \bar{h}_{7} \\
& +1344 \bar{h}_{4}^{2} \bar{h}_{5}^{2} \bar{h}_{7}-1232 \bar{h}_{4} \bar{h}_{5}^{3} \bar{h}_{6} \\
& \left.+231 \bar{h}_{5}^{5}\right) \text {; } \\
& \bar{\alpha}_{00}=2 \sqrt{2}\left(\bar{a}_{10}+\bar{b}_{01}\right), \\
& \bar{\alpha}_{10}=2 \sqrt{2}\left[-\bar{h}_{12}\left(\bar{a}_{10}+\bar{b}_{01}\right)+2 \bar{a}_{20}+\bar{b}_{11}\right], \\
& \bar{\alpha}_{20}=2 \sqrt{2}\left[\left(\bar{a}_{10}+\bar{b}_{01}\right)\left(3 \bar{h}_{03} \bar{h}_{21}-\bar{h}_{22}+\frac{3}{2} \bar{h}_{12}^{2}\right)\right. \\
& -2 \bar{h}_{12} \bar{a}_{20}-\bar{h}_{12} \bar{b}_{11}+3 \bar{a}_{30}+\bar{b}_{21} \\
& \left.-\bar{a}_{11} \bar{h}_{21}-2 \bar{b}_{02} \bar{h}_{21}\right] \text {, } \\
& \bar{\alpha}_{30}=2 \sqrt{2}\left[\left(\bar{a}_{10}+\bar{b}_{01}\right)\right. \\
& \times\left(3 \bar{h}_{13} \bar{h}_{21}+3 \bar{h}_{03} \bar{h}_{31}+3 \bar{h}_{12} \bar{h}_{22}\right. \\
& \left.-15 \bar{h}_{12} \bar{h}_{03} \bar{h}_{21}-\frac{5}{2} \bar{h}_{12}^{3}-\bar{h}_{32}\right) \\
& +\left(3 \bar{a}_{11}+6 \bar{b}_{02}\right) \bar{h}_{12} \bar{h}_{21}-2\left(\bar{b}_{12}+\bar{a}_{21}\right) \bar{h}_{21} \\
& -\left(\bar{a}_{11}+2 \bar{b}_{02}\right) \bar{h}_{31}+4 \bar{a}_{40}+\bar{b}_{31} \\
& +\left(3 \bar{b}_{11}+6 \bar{a}_{20}\right) \bar{h}_{03} \bar{h}_{21} \\
& -\left(2 \bar{a}_{20}+\bar{b}_{11}\right) \bar{h}_{22}+\left(3 \bar{a}_{20}+\frac{3}{2} \bar{b}_{11}\right) \bar{h}_{12}^{2} \\
& \left.-\left(3 \bar{a}_{30}+\bar{b}_{21}\right) \bar{h}_{12}\right], \\
& \bar{\alpha}_{40}=2 \sqrt{2}\left[n_{1}\left(\bar{a}_{10}+\bar{b}_{01}\right)\right. \\
& -\left(\frac{5}{2} \bar{h}_{12}^{3}-3 \bar{h}_{12} \bar{h}_{22}+15 \bar{h}_{12} \bar{h}_{03} \bar{h}_{21}+\bar{h}_{32}\right. \\
& \left.-3 \bar{h}_{13} \bar{h}_{21}-3 \bar{h}_{03} \bar{h}_{31}\right)\left(2 \bar{a}_{20}+\bar{b}_{11}\right) \\
& +\left(\frac{3}{2} \bar{h}_{12}^{2}+3 \bar{h}_{03} \bar{h}_{21}-\bar{h}_{22}\right) \\
& \times\left(3 \bar{a}_{30}+\bar{b}_{21}-\bar{a}_{11} \bar{h}_{21}-2 \bar{b}_{02} \bar{h}_{21}\right) \\
& -\bar{h}_{12}\left(4 \bar{a}_{40}+\bar{b}_{31}-2 \bar{a}_{21} \bar{h}_{21}\right. \\
& -2 \bar{b}_{12} \bar{h}_{21}+2 \bar{a}_{11} \bar{h}_{12} \bar{h}_{21}+4 \bar{b}_{02} \bar{h}_{12} \bar{h}_{21} \\
& \left.\left.-\bar{a}_{11} \bar{h}_{31}-2 \bar{b}_{02} \bar{h}_{31}\right)+n_{3}\right] \text {, } \\
& -\left(\frac{5}{2} \bar{h}_{12}^{3}-3 \bar{h}_{12} \bar{h}_{22}+15 \bar{h}_{12} \bar{h}_{03} \bar{h}_{21}+\bar{h}_{32}\right. \\
& \left.-3 \bar{h}_{13} \bar{h}_{21}-3 \bar{h}_{03} \bar{h}_{31}\right) \\
& \times\left(3 \bar{a}_{30}+\bar{b}_{21}-\bar{a}_{11} \bar{h}_{21}-2 \bar{b}_{02} \bar{h}_{21}\right) \\
& +\left(\frac{3}{2} \bar{h}_{12}^{2}+3 \bar{h}_{03} \bar{h}_{21}-\bar{h}_{22}\right) \\
& \times\left(4 \bar{a}_{40}+\bar{b}_{31}-2 \bar{a}_{21} \bar{h}_{21}\right. \\
& -2 \bar{b}_{12} \bar{h}_{21}+2 \bar{a}_{11} \bar{h}_{12} \bar{h}_{21} \\
& \left.+4 \bar{b}_{02} \bar{h}_{12} \bar{h}_{21}-\bar{a}_{11} \bar{h}_{31}-2 \bar{b}_{02} \bar{h}_{31}\right) \\
& \left.-\bar{h}_{12} n_{3}+n_{4}\right] \text {, } \\
& \bar{\alpha}_{01}=2 \sqrt{2}\left[\frac{2}{3} \bar{a}_{12}+2 \bar{b}_{03}-2 \bar{h}_{03} \bar{a}_{11}-4 \bar{h}_{03} \bar{b}_{02}\right. \\
& \left.+\left(\bar{a}_{10}+\bar{b}_{01}\right)\left(5 \bar{h}_{03}^{2}-2 \bar{h}_{04}\right)\right], \\
& \bar{\alpha}_{11}=2 \sqrt{2}\left[( \overline { a } _ { 1 0 } + \overline { b } _ { 0 1 } ) \left(10 \bar{h}_{04} \bar{h}_{12}-2 \bar{h}_{14}\right.\right. \\
& \left.+10 \bar{h}_{03} \bar{h}_{13}-35 \bar{h}_{12} \bar{h}_{03}^{2}\right) \\
& +\left(\bar{a}_{11}+2 \bar{b}_{02}\right)\left(-2 \bar{h}_{13}+10 \bar{h}_{12} \bar{h}_{03}\right) \\
& -2 \bar{h}_{12}\left(\bar{a}_{12}+3 \bar{b}_{03}\right) \\
& +\left(2 \bar{a}_{20}+\bar{b}_{11}\right)\left(5 \bar{h}_{03}^{2}-2 \bar{h}_{04}\right) \\
& \left.-4 \bar{h}_{03}\left(\bar{b}_{12}+\bar{a}_{21}\right)+\frac{4}{3} \bar{a}_{22}+2 \bar{b}_{13}\right] ; \\
& \bar{h}_{4}=-\frac{1}{2} \bar{h}_{21}^{2}+\bar{h}_{40}, \quad \bar{h}_{5}=\bar{h}_{12} \bar{h}_{21}^{2}-\bar{h}_{21} \bar{h}_{31}+\bar{h}_{50}, \\
& \bar{h}_{6}=-2 \bar{h}_{12}^{2} \bar{h}_{21}^{2}-\bar{h}_{03} \bar{h}_{21}^{3}+\bar{h}_{21}^{2} \bar{h}_{22} \\
& +2 \bar{h}_{12} \bar{h}_{21} \bar{h}_{31}-\frac{1}{2} \bar{h}_{31}^{2}-\bar{h}_{21} \bar{h}_{41}+\bar{h}_{60}, \\
& \bar{h}_{7}=-4 \bar{h}_{22} \bar{h}_{21}^{2} \bar{h}_{12}+\bar{h}_{70}-\bar{h}_{51} \bar{h}_{21}-\bar{h}_{13} \bar{h}_{21}^{3} \\
& +\bar{h}_{32} \bar{h}_{21}^{2}+4 \bar{h}_{12}^{3} \bar{h}_{21}^{2}-\bar{h}_{31} \bar{h}_{41}+\bar{h}_{12} \bar{h}_{31}^{2} \\
& +2 \bar{h}_{22} \bar{h}_{21} \bar{h}_{31}+6 \bar{h}_{03} \bar{h}_{21}^{3} \bar{h}_{12}-3 \bar{h}_{03} \bar{h}_{21}^{2} \bar{h}_{31} \\
& -4 \bar{h}_{12}^{2} \bar{h}_{21} \bar{h}_{31}+2 \bar{h}_{12} \bar{h}_{21} \bar{h}_{41} \text {, }
\end{aligned}
$$




$$
\begin{aligned}
& \bar{h}_{8}=\bar{h}_{80}-\bar{h}_{23} \bar{h}_{21}^{3}-\bar{h}_{61} \bar{h}_{21}+\bar{h}_{04} \bar{h}_{21}^{4}+\bar{h}_{42} \bar{h}_{21}^{2} \\
& -2 \bar{h}_{12}^{2} \bar{h}_{31}^{2}-8 \bar{h}_{12}^{4} \bar{h}_{21}^{2}-2 \bar{h}_{22}^{2} \bar{h}_{21}^{2} \\
& -\frac{9}{2} \bar{h}_{03}^{2} \bar{h}_{21}^{4}-\bar{h}_{31} \bar{h}_{51}+\bar{h}_{22} \bar{h}_{31}^{2}-\frac{1}{2} \bar{h}_{41}^{2} \\
& +8 \bar{h}_{12}^{3} \bar{h}_{21} \bar{h}_{31}-4 \bar{h}_{12}^{2} \bar{h}_{21} \bar{h}_{41}+12 \bar{h}_{22} \bar{h}_{21}^{2} \bar{h}_{12}^{2} \\
& -24 \bar{h}_{03} \bar{h}_{21}^{3} \bar{h}_{12}^{2}+2 \bar{h}_{12} \bar{h}_{31} \bar{h}_{41}+6 \bar{h}_{12} \bar{h}_{21}^{3} \bar{h}_{13} \\
& -4 \bar{h}_{12} \bar{h}_{21}^{2} \bar{h}_{32}+2 \bar{h}_{12} \bar{h}_{21} \bar{h}_{51}+6 \bar{h}_{22} \bar{h}_{21}^{3} \bar{h}_{03} \\
& +2 \bar{h}_{22} \bar{h}_{21} \bar{h}_{41}+2 \bar{h}_{32} \bar{h}_{21} \bar{h}_{31}-3 \bar{h}_{03} \bar{h}_{21} \bar{h}_{31}^{2} \\
& -3 \bar{h}_{03} \bar{h}_{21}^{2} \bar{h}_{41}-3 \bar{h}_{13} \bar{h}_{21}^{2} \bar{h}_{31} \\
& -8 \bar{h}_{12} \bar{h}_{22} \bar{h}_{21} \bar{h}_{31}+18 \bar{h}_{12} \bar{h}_{03} \bar{h}_{21}^{2} \bar{h}_{31} \text {, } \\
& \bar{h}_{9}=\bar{h}_{12}\left[\bar{h}_{21}^{4}\left(45 \bar{h}_{03}^{2}-8 \bar{h}_{04}\right)+6 \bar{h}_{21}^{3}\left(\bar{h}_{23}-8 \bar{h}_{03} \bar{h}_{22}\right)\right. \\
& +2 \bar{h}_{21}^{2}\left(9 \bar{h}_{13} \bar{h}_{31}-2 \bar{h}_{42}+6 \bar{h}_{22}^{2}+9 \bar{h}_{03} \bar{h}_{41}\right) \\
& +2 \bar{h}_{21}\left(9 \bar{h}_{03} \bar{h}_{31}^{2}-4 \bar{h}_{41} \bar{h}_{22}-4 \bar{h}_{31} \bar{h}_{32}\right) \\
& \left.-4 \bar{h}_{22} \bar{h}_{31}^{2}+\bar{h}_{41}^{2}+2 \bar{h}_{31} \bar{h}_{51}+2 \bar{h}_{21} \bar{h}_{61}\right] \\
& +4 \bar{h}_{12}^{2}\left[\overline { h } _ { 2 1 } \left(3 \bar{h}_{21} \bar{h}_{32}-6 \bar{h}_{21}^{2} \bar{h}_{13}-\bar{h}_{51}\right.\right. \\
& \left.\left.+6 \bar{h}_{31} \bar{h}_{22}-18 \bar{h}_{31} \bar{h}_{03} \bar{h}_{21}\right)-\bar{h}_{31} \bar{h}_{41}\right] \\
& +4 \bar{h}_{12}^{3}\left(20 \bar{h}_{03} \bar{h}_{21}^{3}+2 \bar{h}_{41} \bar{h}_{21}-8 \bar{h}_{22} \bar{h}_{21}^{2}+\bar{h}_{31}^{2}\right) \\
& +\bar{h}_{21}\left(2 \bar{h}_{42} \bar{h}_{31}-16 \bar{h}_{12}^{4} \bar{h}_{31}-4 \bar{h}_{22}^{2} \bar{h}_{31}+2 \bar{h}_{22} \bar{h}_{51}\right. \\
& \left.+2 \bar{h}_{32} \bar{h}_{41}-3 \bar{h}_{13} \bar{h}_{31}^{2}-\bar{h}_{71}-6 \bar{h}_{03} \bar{h}_{31} \bar{h}_{41}\right) \\
& +\bar{h}_{21}^{2}\left(16 \bar{h}_{12}^{5}-4 \bar{h}_{32} \bar{h}_{22}-3 \bar{h}_{31} \bar{h}_{23}\right. \\
& \left.-3 \bar{h}_{03} \bar{h}_{51}+\bar{h}_{52}+18 \bar{h}_{22} \bar{h}_{31} \bar{h}_{03}\right) \\
& +2 \bar{h}_{22} \bar{h}_{31} \bar{h}_{41}+6 \bar{h}_{32} \bar{h}_{21}^{3} \bar{h}_{03}+4 \bar{h}_{04} \bar{h}_{21}^{3} \bar{h}_{31} \\
& -\bar{h}_{41} \bar{h}_{51}-\bar{h}_{31} \bar{h}_{61}+\bar{h}_{32} \bar{h}_{31}^{2}-\bar{h}_{03} \bar{h}_{31}^{3} \\
& -9 \bar{h}_{03} \bar{h}_{21}^{4} \bar{h}_{13}-18 \bar{h}_{31} \bar{h}_{03}^{2} \bar{h}_{21}^{3}+\bar{h}_{14} \bar{h}_{21}^{4} \\
& -\bar{h}_{33} \bar{h}_{21}^{3}+6 \bar{h}_{22} \bar{h}_{21}^{3} \bar{h}_{13}+\bar{h}_{90} \text {; } \\
& n_{1}=\frac{1}{8}\left(12 \bar{h}_{22}^{2}+35 \bar{h}_{12}^{4}+24 \bar{h}_{12} \bar{h}_{32}\right. \\
& -60 \bar{h}_{12}^{2} \bar{h}_{22}-72 \bar{h}_{12} \bar{h}_{03} \bar{h}_{31}+324 \bar{h}_{12}^{2} \bar{h}_{03} \bar{h}_{21} \\
& \left.+108 \bar{h}_{03}^{2} \bar{h}_{21}^{2}-72 \bar{h}_{12} \bar{h}_{13} \bar{h}_{21}-72 \bar{h}_{22} \bar{h}_{03} \bar{h}_{21}\right),
\end{aligned}
$$

$$
n_{2}=-\frac{1}{8}\left(-24 \bar{h}_{22} \bar{h}_{32}+60 \bar{h}_{12} \bar{h}_{22}^{2}+60 \bar{h}_{12}^{2} \bar{h}_{32}\right.
$$$$
-140 \bar{h}_{12}^{3} \bar{h}_{22}+63 \bar{h}_{12}^{5}
$$$$
-180 \bar{h}_{12}^{2} \bar{h}_{03} \bar{h}_{31}+780 \bar{h}_{12}^{3} \bar{h}_{03} \bar{h}_{21}
$$$$
+72 \bar{h}_{22} \bar{h}_{03} \bar{h}_{31}-504 \bar{h}_{12} \bar{h}_{22} \bar{h}_{03} \bar{h}_{21}
$$$$
-216 \bar{h}_{03}^{2} \bar{h}_{21} \bar{h}_{31}+972 \bar{h}_{12} \bar{h}_{03}^{2} \bar{h}_{21}^{2}
$$$$
+72 \bar{h}_{03} \bar{h}_{21} \bar{h}_{32}+72 \bar{h}_{22} \bar{h}_{13} \bar{h}_{21}
$$$$
\left.-180 \bar{h}_{12}^{2} \bar{h}_{13} \bar{h}_{21}-216 \bar{h}_{03} \bar{h}_{21}^{2} \bar{h}_{13}\right),
$$$$
n_{3}=5 \bar{a}_{50}+\bar{b}_{41}-2 \bar{b}_{22} \bar{h}_{21}-\bar{a}_{11} \bar{h}_{41}
$$$$
+2 \bar{a}_{11} \bar{h}_{22} \bar{h}_{21}-3 \bar{a}_{11} \bar{h}_{03} \bar{h}_{21}^{2}+2 \bar{a}_{11} \bar{h}_{12} \bar{h}_{31}
$$$$
-4 \bar{a}_{11} \bar{h}_{12}^{2} \bar{h}_{21}-2 \bar{b}_{02} \bar{h}_{41}+4 \bar{b}_{02} \bar{h}_{22} \bar{h}_{21}
$$$$
-6 \bar{b}_{02} \bar{h}_{03} \bar{h}_{21}^{2}+4 \bar{b}_{02} \bar{h}_{12} \bar{h}_{31}-8 \bar{b}_{02} \bar{h}_{12}^{2} \bar{h}_{21}
$$$$
-2 \bar{b}_{12} \bar{h}_{31}+4 \bar{b}_{12} \bar{h}_{12} \bar{h}_{21}-2 \bar{a}_{21} \bar{h}_{31}
$$$$
+4 \bar{a}_{21} \bar{h}_{12} \bar{h}_{21}-3 \bar{a}_{31} \bar{h}_{21}+3 \bar{b}_{03} \bar{h}_{21}^{2}+\bar{a}_{12} \bar{h}_{21}^{2} \text {, }
$$

$$
n_{4}=4 \bar{b}_{12} \bar{h}_{22} \bar{h}_{21}+4 \bar{b}_{02} \bar{h}_{32} \bar{h}_{21}+6 \bar{a}_{31} \bar{h}_{12} \bar{h}_{21}
$$$$
+2 \bar{a}_{11} \bar{h}_{12} \bar{h}_{41}+4 \bar{b}_{12} \bar{h}_{12} \bar{h}_{31}-4 \bar{a}_{11} \bar{h}_{12}^{2} \bar{h}_{31}
$$$$
+4 \bar{b}_{02} \bar{h}_{12} \bar{h}_{41}-12 \bar{b}_{03} \bar{h}_{21}^{2} \bar{h}_{12}-4 \bar{a}_{12} \bar{h}_{21}^{2} \bar{h}_{12}
$$$$
+2 \bar{a}_{11} \bar{h}_{22} \bar{h}_{31}+6 \bar{b}_{03} \bar{h}_{21} \bar{h}_{31}-3 \bar{a}_{11} \bar{h}_{13} \bar{h}_{21}^{2}
$$$$
+6 \bar{a}_{60}+\bar{b}_{51}-6 \bar{b}_{12} \bar{h}_{03} \bar{h}_{21}^{2}+2 \bar{a}_{11} \bar{h}_{32} \bar{h}_{21}
$$$$
+4 \bar{b}_{02} \bar{h}_{22} \bar{h}_{31}+4 \bar{a}_{21} \bar{h}_{22} \bar{h}_{21}+2 \bar{a}_{12} \bar{h}_{21} \bar{h}_{31}
$$$$
+4 \bar{b}_{22} \bar{h}_{12} \bar{h}_{21}-8 \bar{b}_{12} \bar{h}_{12}^{2} \bar{h}_{21}+16 \bar{b}_{02} \bar{h}_{12}^{3} \bar{h}_{21}
$$$$
+8 \bar{a}_{11} \bar{h}_{12}^{3} \bar{h}_{21}-6 \bar{b}_{02} \bar{h}_{13} \bar{h}_{21}^{2}-8 \bar{a}_{21} \bar{h}_{12}^{2} \bar{h}_{21}
$$$$
-8 \bar{b}_{02} \bar{h}_{12}^{2} \bar{h}_{31}-4 \bar{a}_{41} \bar{h}_{21}-2 \bar{b}_{32} \bar{h}_{21}
$$$$
-6 \bar{a}_{21} \bar{h}_{03} \bar{h}_{21}^{2}+4 \bar{a}_{21} \bar{h}_{12} \bar{h}_{31}+3 \bar{b}_{13} \bar{h}_{21}^{2}
$$$$
+2 \bar{a}_{22} \bar{h}_{21}^{2}-6 \bar{a}_{11} \bar{h}_{03} \bar{h}_{21} \bar{h}_{31}+18 \bar{a}_{11} \bar{h}_{03} \bar{h}_{21}^{2} \bar{h}_{12}
$$$$
-8 \bar{a}_{11} \bar{h}_{12} \bar{h}_{22} \bar{h}_{21}-12 \bar{b}_{02} \bar{h}_{03} \bar{h}_{21} \bar{h}_{31}+36 \bar{b}_{02} \bar{h}_{03} \bar{h}_{21}^{2} \bar{h}_{12}
$$$$
-16 \bar{b}_{02} \bar{h}_{12} \bar{h}_{22} \bar{h}_{21}-2 \bar{b}_{22} \bar{h}_{31}-\bar{a}_{11} \bar{h}_{51}-2 \bar{b}_{12} \bar{h}_{41}
$$$$
-2 \bar{a}_{21} \bar{h}_{41}-2 \bar{b}_{02} \bar{h}_{51}-3 \bar{a}_{31} \bar{h}_{31} \text {. }
$$ 
For convenience, let

$$
\begin{gathered}
L^{*}=L_{1} \cup L_{2}, \quad L_{1}^{*}=L^{*} \cap U_{1}, \\
L_{2}^{*}=L^{*} \cap U_{2}, \quad L_{3}^{*}=\mathrm{Cl} .\left(L^{*}-\bigcup_{i=1}^{2} L_{i}^{*}\right), \\
L_{0}^{1}=L_{1}^{*},
\end{gathered}
$$

$L_{0} \cap U_{2}=L_{0}^{2} \cup L_{0}^{3} \quad($ as shown in Figure 2(b)),

$$
L_{0}^{4}=\mathrm{Cl} .\left(L_{0}-\bigcup_{j=1}^{3} L_{0}^{j}\right) .
$$

Theorem 3. Assume that system (1) has a compound loop $L_{0}$ as stated before. Then, the functions $M_{i}(h, \delta)$ given in (3) at $h=0$ have the following expansions:

$$
\begin{aligned}
M_{1}(h, \delta)= & c_{0}(\delta)+c_{1}(\delta)|h|^{3 / 4}+B_{00} c_{2}(\delta)|h|^{5 / 6} \\
& -c_{3}(\delta) h \ln |h|+c_{4}(\delta) h+B_{10} c_{5}(\delta)|h|^{7 / 6} \\
& +c_{6}(\delta)|h|^{5 / 4}+c_{7}(\delta)|h|^{7 / 4} \\
& -\frac{1}{11} B_{00} c_{8}(\delta)|h|^{11 / 6}-c_{9}(\delta) h^{2} \ln |h|+O\left(h^{2}\right),
\end{aligned}
$$

$$
\begin{aligned}
M_{2}(h, \delta)= & \bar{c}_{0}(\delta)+c_{1}(\delta)|h|^{3 / 4}+c_{3}(\delta) h \ln |h| \\
& +\bar{c}_{3}(\delta) h+c_{6}(\delta)|h|^{5 / 4}+c_{7}(\delta)|h|^{7 / 4} \\
& +c_{9}(\delta) h^{2} \ln |h|+O\left(h^{2}\right)
\end{aligned}
$$

for $0<-h \ll 1$, and

$$
\begin{aligned}
M_{3}(h, \delta)= & \widetilde{c}_{0}(\delta)-D_{1} c_{1}(\delta) h^{3 / 4}+B_{00}^{*} c_{2}(\delta) h^{5 / 6} \\
& +\widetilde{c}_{2}(\delta) h+B_{10}^{*} c_{5}(\delta) h^{7 / 6}-D_{2} c_{6}(\delta) h^{5 / 4} \\
& +D_{1} c_{7}(\delta) h^{7 / 4}+\frac{1}{11} B_{00}^{*} c_{8}(\delta) h^{11 / 6}+O\left(h^{2}\right)
\end{aligned}
$$

for $0<h \ll 1$, where

$$
\begin{array}{ll}
c_{1}(\delta)=c_{1}\left(S_{2}, \delta\right), & c_{2}(\delta)=c_{1}\left(S_{1}, \delta\right), \\
c_{3}(\delta)=c_{2}\left(S_{2}, \delta\right), & c_{5}(\delta)=c_{3}\left(S_{1}, \delta\right), \\
c_{6}(\delta)=c_{4}\left(S_{2}, \delta\right), & c_{7}(\delta)=c_{5}\left(S_{2}, \delta\right), \\
c_{8}(\delta)=c_{4}\left(S_{1}, \delta\right), & c_{9}(\delta)=c_{6}\left(S_{2}, \delta\right),
\end{array}
$$

$$
\begin{gathered}
c_{0}(\delta)=M_{1}(0, \delta)=\oint_{L^{*}} q d x-p d y=\sum_{i=1}^{2} \int_{L_{i}} q d x-p d y, \\
\bar{c}_{0}(\delta)=M_{2}(0, \delta)=\oint_{L_{3}} q d x-p d y, \\
\widetilde{c}_{0}(\delta)=M_{3}(0, \delta)=c_{0}(\delta)+\bar{c}_{0}(\delta),
\end{gathered}
$$$$
c_{4}(\delta)=\int_{L_{1}^{*}}\left(p_{x}+q_{y}-\sigma\right) d t
$$$$
+\int_{L_{2}^{*}}\left[p_{x}+q_{y}-\eta_{0}-\eta_{1}\left(x-x_{2}\right)\right] d t
$$$$
+\int_{L_{3}^{*}}\left(p_{x}+q_{y}\right) d t+t_{2} c_{1}(\delta)+t_{3} c_{2}(\delta)+t_{4} c_{3}(\delta) \text {, }
$$$$
\bar{c}_{3}(\delta)=\oint_{L_{3}}\left[p_{x}+q_{y}-\eta_{0}-\eta_{1}\left(x-x_{2}\right)\right] d t
$$$$
+t_{0} c_{1}(\delta)+t_{1} c_{3}(\delta)
$$

$$
\begin{aligned}
\widetilde{c}_{2}(\delta)= & \int_{L_{1}^{*}}\left(p_{x}+q_{y}-\sigma\right) d t+\int_{L_{0}^{2} \cup L_{0}^{3}}\left(p_{x}+q_{y}-\eta_{0}\right) d t \\
& +\int_{L_{0}^{4}}\left(p_{x}+q_{y}\right) d t+t_{5} c_{1}(\delta)+t_{6} c_{2}(\delta),
\end{aligned}
$$

where $\sigma=\left.\left(p_{x}+q_{y}\right)\right|_{\left(x_{1}, y_{1}\right)}, \quad \eta_{0}=\left.\left(p_{x}+q_{y}\right)\right|_{\left(x_{2}, y_{2}\right)}, \eta_{1}=\left(p_{x x}+\right.$ $\left.q_{y x}\right)\left.\right|_{\left(x_{2}, y_{2}\right)}$. In particular,

$$
\begin{gathered}
c_{4}(\delta)=\sum_{i=1}^{2} \int_{L_{i}}\left(p_{x}+q_{y}\right) d t, \quad \bar{c}_{3}(\delta)=\oint_{L_{3}}\left(p_{x}+q_{y}\right) d t \\
\widetilde{c}_{2}(\delta)=\sum_{i=1}^{3} \int_{L_{i}}\left(p_{x}+q_{y}\right) d t
\end{gathered}
$$

if $c_{1}(\delta)=c_{2}(\delta)=c_{3}(\delta)=0$. Here, $t_{i}, i=0,1, \ldots, 6$, are constants and $B_{00}, B_{00}^{*}, B_{10}, B_{10}^{*}$ are given in Lemma 1 .

Proof. First, by (6), (10) with $i=2$, (12), (14), (29), and Theorem 2.2 in [4], we directly obtain $(27)$ with $\bar{c}_{0}(\delta), \bar{c}_{3}(\delta)$ given by (30) and (31), respectively. Then we study the expansions of $M_{1}$ and $M_{3}$.

By (5), (7), (29), and Lemmas 1 and 2, we have

$$
\begin{aligned}
M_{1}(h, \delta)= & c_{1}(\delta)|h|^{3 / 4}+B_{00} c_{2}(\delta)|h|^{5 / 6}-c_{3}(\delta) h \ln |h| \\
& +B_{10} c_{5}(\delta)|h|^{7 / 6}+c_{6}(\delta)|h|^{5 / 4} \\
& +c_{7}(\delta)|h|^{7 / 4}-\frac{1}{11} B_{00} c_{8}(\delta)|h|^{11 / 6} \\
& -c_{9}(\delta) h^{2} \ln |h|+O\left(h^{2}\right)+N(h, \delta)
\end{aligned}
$$


for $0<-h \ll 1$, and

$$
\begin{aligned}
M_{3}(h, \delta)= & -D_{1} c_{1}(\delta) h^{3 / 4}+B_{00}^{*} c_{2}(\delta) h^{5 / 6}+B_{10}^{*} c_{5}(\delta) h^{7 / 6} \\
& -D_{2} c_{6}(\delta) h^{5 / 4}+D_{1} c_{7}(\delta) h^{7 / 4} \\
& +\frac{1}{11} B_{00}^{*} c_{8}(\delta) h^{11 / 6}+O\left(h^{2}\right)+N^{*}(h, \delta)
\end{aligned}
$$

for $0<h \ll 1$, where

$$
\begin{aligned}
N(h, \delta) & =N_{11}(h, \delta)+N_{12}(h, \delta)+I_{13}(h, \delta), \\
N^{*}(h, \delta) & =N_{31}(h, \delta)+N_{32}(h, \delta)+N_{33}(h, \delta)+I_{34}(h, \delta) .
\end{aligned}
$$

Let

$$
\begin{aligned}
& N(h, \delta)=c_{0}(\delta)+c_{4}(\delta) h+O\left(h^{2}\right), \\
& N^{*}(h, \delta)=\widetilde{c}_{0}(\delta)+\widetilde{c}_{2}(\delta) h+O\left(h^{2}\right) .
\end{aligned}
$$

It follows further that

$$
\begin{aligned}
& c_{0}(\delta)=N_{11}(0, \delta)+N_{12}(0, \delta)+I_{13}(0, \delta) \\
& =\lim _{\epsilon_{0} \rightarrow 0}\left[N_{11}(0, \delta)+N_{12}(0, \delta)+I_{13}(0, \delta)\right] \\
& =\lim _{\epsilon_{0} \rightarrow 0} I_{13}(0, \delta)=\oint_{L_{1} \cup L_{2}} q d x-p d y \\
& =\sum_{i=1}^{2} \int_{L_{i}} q d x-p d y=M_{1}(0, \delta), \\
& \widetilde{\mathcal{c}}_{0}(\delta)=N_{31}(0, \delta)+N_{32}(0, \delta)+N_{33}(0, \delta)+I_{34}(0, \delta) \\
& =\lim _{\epsilon_{0} \rightarrow 0}\left[N_{31}(0, \delta)+N_{32}(0, \delta)\right. \\
& \left.+N_{33}(0, \delta)+I_{34}(0, \delta)\right] \\
& =\lim _{\epsilon_{0} \rightarrow 0} I_{34}(0, \delta)=\oint_{L_{0}} q d x-p d y \\
& =\sum_{i=1}^{3} \int_{L_{i}} q d x-p d y=M_{3}(0, \delta), \\
& c_{4}(\delta)+O(h)=N_{h}(h, \delta)=M_{1 h}(h, \delta) \\
& +\frac{3}{4} c_{1}(\delta)|h|^{-1 / 4}+\frac{5}{6} B_{00} c_{2}(\delta)|h|^{-1 / 6} \\
& +c_{3}(\delta)(\ln |h|+1)+O\left(|h|^{1 / 6}\right), \\
& \widetilde{c}_{2}(\delta)+O(h)=N_{h}^{*}(h, \delta) \\
& \begin{aligned}
= & M_{3 h}(h, \delta)+\frac{3}{4} D_{1} c_{1}(\delta) h^{-1 / 4} \\
& -\frac{5}{6} B_{00}^{*} c_{2}(\delta) h^{-1 / 6}+O\left(h^{1 / 6}\right) .
\end{aligned}
\end{aligned}
$$

Then by Lemma 3.1.2 in [5], we have

$$
\begin{aligned}
c_{4}(\delta)= & N_{h}(0, \delta) \\
= & \lim _{h \rightarrow 0^{-}}\left[\oint_{L_{h 1}}\left(p_{x}+q_{y}\right) d t+\frac{3}{4} c_{1}(\delta)|h|^{-1 / 4}\right. \\
& \left.+\frac{5}{6} B_{00} c_{2}(\delta)|h|^{-1 / 6}+c_{3}(\delta)(\ln |h|+1)\right], \\
\widetilde{c}_{2}(\delta)= & N_{h}^{*}(0, \delta) \quad \\
= & \lim _{h \rightarrow 0^{+}}\left[\oint_{L_{h 3}}\left(p_{x}+q_{y}\right) d t\right. \\
& \left.+\frac{3}{4} D_{1} c_{1}(\delta) h^{-1 / 4}-\frac{5}{6} B_{00}^{*} c_{2}(\delta) h^{-1 / 6}\right] .
\end{aligned}
$$

It is easy to see that

$$
\begin{aligned}
& \oint_{L_{h 1}}\left(p_{x}+q_{y}\right) d t= \sum_{i=1}^{3} \int_{L_{h 1}^{(i)}}\left(p_{x}+q_{y}\right) d t \\
&= \int_{L_{h 1}^{(1)}}\left(p_{x}+q_{y}-\sigma\right) d t \\
&+\int_{L_{h 1}^{(2)}}\left[p_{x}+q_{y}-\eta_{0}\right. \\
&+\int_{L_{h 1}^{(3)}}\left(p_{x}+q_{y}\right) d t+\sigma \int_{L_{h 1}^{(1)}} d t \\
&+\eta_{0} \int_{L_{h 1}^{(2)}} d t+\eta_{1} \int_{L_{h 1}^{(2)}}\left(x-x_{2}\right) d t, \\
& \oint_{L_{h 3}}\left(p_{x}+q_{y}\right) d t \\
&+\sum_{i=1}^{4} \int_{L_{h 3}^{(i)}}\left(p_{x}+q_{y}\right) d t=\int_{L_{h 3}^{(1)}}\left(p_{x}+q_{y}-\sigma\right) d t \\
&+\int_{L_{h 3}^{(2)} \cup L_{h 3}^{(3)}}\left(p_{x}+q_{y}-\eta_{0}\right) d t+\int_{L_{h 3}^{(4)}}\left(p_{x}+q_{y}\right) d t \\
&+\sigma \int_{L_{h 3}^{(1)}} d t+\eta_{0} \int_{L_{h 3}^{(2)} \cup L_{h 3}^{(3)}} d t .
\end{aligned}
$$

Noting $\widetilde{h}_{3}<0, \bar{h}_{4}<0$, by (19), (23), and (29), we have

$$
\begin{aligned}
c_{4}(\delta)=\lim _{h \rightarrow 0^{-}}\left[\int_{L_{h 1}^{(1)}}\left(p_{x}+q_{y}-\sigma\right) d t\right. \\
\quad+\int_{L_{h 1}^{(2)}}\left[p_{x}+q_{y}-\eta_{0}-\eta_{1}\left(x-x_{2}\right)\right] d t \\
\left.\quad+\int_{L_{h 1}^{(3)}}\left(p_{x}+q_{y}\right) d t\right]
\end{aligned}
$$




$$
\begin{gathered}
+\lim _{h \rightarrow 0^{-}} \sigma\left[\int_{L_{h 1}^{(1)}} d t-\frac{5 \sqrt{2}}{3} B_{00}\left|\widetilde{h}_{3}\right|^{-1 / 3}|h|^{-1 / 6}\right] \\
+\lim _{h \rightarrow 0^{-}} \eta_{0}\left[\int_{L_{h 1}^{(2)}} d t+\frac{3 \sqrt{2}}{2} \widetilde{A}_{0}\left|\bar{h}_{4}\right|^{-1 / 4}|h|^{-1 / 4}\right. \\
+O(1)(\ln |h|+1)] \\
+\lim _{h \rightarrow 0^{-}} \eta_{1}\left[\int_{L_{h 1}^{(2)}}\left(x-x_{2}\right) d t\right. \\
+\widetilde{c}_{2}(\delta)=\lim _{h \rightarrow 0^{+}}\left[\int_{L_{h 3}^{(1)}}\left(p_{x}+q_{y}-\sigma\right) d t\right. \\
+\int_{L_{h 3}^{(2)} \cup L_{h 3}^{(3)}}\left(p_{x}+q_{y}-\eta_{0}\right) d t \\
\left.+\int_{L_{h 3}^{(4)}}\left(p_{x}+q_{y}\right) d t\right] \\
\left.+\left.\lim _{h \rightarrow 0^{+}} \sigma\left|\int_{L_{h 3}^{(1)}} d t+\frac{5 \sqrt{2}}{3} B_{00}^{*}\right| \widetilde{h}_{3}\right|^{-1 / 3} h^{-1 / 6}\right] \\
+\lim _{h \rightarrow 0^{+}} \eta_{0}\left[\int_{L_{h 3}^{(2)} \cup L_{h 3}^{(3)}} d t-3 \sqrt{2} \bar{A}_{0}\left|\bar{h}_{4}\right|^{-1 / 4} h^{-1 / 4}\right] .
\end{gathered}
$$

Then by the proof of (3.13) in [3], the following equations hold:

$$
\begin{gathered}
\lim _{h \rightarrow 0^{-}}\left[\int_{L_{h 1}^{(1)}} d t-\frac{5 \sqrt{2}}{3} B_{00}\left|\widetilde{h}_{3}\right|^{-1 / 3}|h|^{-1 / 6}\right]=T_{0}, \\
\lim _{h \rightarrow 0^{+}}\left[\int_{L_{h 3}^{(1)}} d t+\frac{5 \sqrt{2}}{3} B_{00}^{*}\left|\widetilde{h}_{3}\right|^{-1 / 3} h^{-1 / 6}\right]=T_{0}^{*}, \\
\lim _{h \rightarrow 0^{-}} \int_{L_{h 1}^{(1)}}\left(p_{x}+q_{y}-\sigma\right) d t=\lim _{h \rightarrow 0^{+}} \int_{L_{h 3}^{(1)}}\left(p_{x}+q_{y}-\sigma\right) d t \\
=\int_{L_{1}^{*}}\left(p_{x}+q_{y}-\sigma\right) d t .
\end{gathered}
$$

Here, $T_{0}$ and $T_{0}^{*}$ are constants. By a similar argument used in Theorems 2.2 and 2.4 in [4], one can obtain

$$
\begin{aligned}
& \lim _{h \rightarrow 0^{-}}\left[\int_{L_{h 1}^{(2)}} d t+\frac{3 \sqrt{2}}{2} \widetilde{A}_{0}\left|\bar{h}_{4}\right|^{-1 / 4}|h|^{-1 / 4}+O(1)(\ln |h|+1)\right] \\
& \quad=T_{1}, \\
& \lim _{h \rightarrow 0^{-}}\left[\int_{L_{h 1}^{(2)}}\left(x-x_{2}\right) d t-\frac{\sqrt{2}}{4}\left|\bar{h}_{4}\right|^{-1 / 2}(\ln |h|+1)\right]=T_{1}^{*}, \\
& \lim _{h \rightarrow 0^{+}}\left[\int_{L_{h 3}^{(i)}} d t-\frac{3 \sqrt{2}}{2} \bar{A}_{0}\left|\bar{h}_{4}\right|^{-1 / 4} h^{-1 / 4}\right]=T_{i}, \quad i=2,3,
\end{aligned}
$$

$$
\begin{gathered}
\lim _{h \rightarrow 0^{-}} \int_{L_{h 1}^{(2)}}\left[p_{x}+q_{y}-\eta_{0}-\eta_{1}\left(x-x_{2}\right)\right] d t \\
=\int_{L_{2}^{*}}\left[p_{x}+q_{y}-\eta_{0}-\eta_{1}\left(x-x_{2}\right)\right] d t, \\
\lim _{h \rightarrow 0^{+}} \int_{L_{h 3}^{(i)}}\left(p_{x}+q_{y}-\eta_{0}\right) d t=\int_{L_{0}^{i}}\left(p_{x}+q_{y}-\eta_{0}\right) d t, \\
i=2,3 .
\end{gathered}
$$

Here $T_{1}^{*}, T_{i}, i=1,2,3$, are constants. Therefore, we can obtain (31) and (32). Thus we have proved Theorem 3.

In the following we use Theorem 3 to study the problem of limit cycle bifurcation near $L_{0}$. For the sake of convenience, we say that (1) has a distribution $(i, j)+k$ of $i+j+k$ limit cycles if there are $i$ and $j$ limit cycles near the inside of $L^{*}$ and $L_{3}$, respectively, and $k$ limit cycles near the outside of $L_{0}$. Then we can prove the following theorem.

Theorem 4. Assume that system (1) has a compound loop $L_{0}$ as stated before and (26)-(28) hold. Define $c_{4}^{*}(\delta)=$ $\left.c_{4}(\delta)\right|_{\mathcal{1}_{1}=c_{2}=c_{3}=0}, \bar{c}_{3}^{*}(\delta)=\left.\bar{c}_{3}(\delta)\right|_{c_{1}=c_{3}=0}, \widetilde{c}_{2}^{*}(\delta)=c_{4}^{*}(\delta)+$ $\bar{c}_{3}^{*}(\delta), c_{3}^{*}(\delta)=\left.c_{3}(\delta)\right|_{c_{1}=0}$. Let there exist $\delta_{0} \in \mathbb{R}^{m}$, such that $\left(c_{0}, \bar{c}_{0}, c_{1}, c_{2}, c_{3}^{*}, c_{4}^{*}, \bar{c}_{3}^{*}\right)\left(\delta_{0}\right)=(0,0,0,0,0,0,0)$.

(1) If $c_{l}\left(\delta_{0}\right) \neq 0, c_{j}\left(\delta_{0}\right)=0, j=5, \ldots, l-1$, and

$\operatorname{rank} \frac{\partial\left(c_{0}, \bar{c}_{0}, c_{1}, c_{2}, c_{3}^{*}, c_{4}^{*}, \bar{c}_{3}^{*}, c_{5}, \ldots, c_{l-1}\right)}{\partial\left(\delta_{1}, \delta_{2}, \delta_{3}, \delta_{4}, \delta_{5}, \delta_{6}, \delta_{7}, \delta_{8}, \ldots, \delta_{m}\right)}=l+2$,

then (1) can have $2 l+1$ limit cycles near $L_{0}$ for some $(\epsilon, \delta)$ near $\left(0, \delta_{0}\right)$, where $l=6,7$ or 9 .

(2) If $c_{5}\left(\delta_{0}\right) c_{6}\left(\delta_{0}\right)<0$, and

$$
\operatorname{rank} \frac{\partial\left(c_{0}, \bar{c}_{0}, c_{1}, c_{2}, c_{3}^{*}, c_{4}^{*}, \bar{c}_{3}^{*}\right)}{\partial\left(\delta_{1}, \delta_{2}, \delta_{3}, \delta_{4}, \ldots, \delta_{m}\right)}=7,
$$

then (1) can have 11 limit cycles near $L_{0}$ for some $(\epsilon, \delta)$ near $\left(0, \delta_{0}\right)$.

(3) If $c_{8}\left(\delta_{0}\right) c_{9}\left(\delta_{0}\right)>0, c_{j}\left(\delta_{0}\right)=0, j=5,6,7$, and

$\operatorname{rank} \frac{\partial\left(c_{0}, \bar{c}_{0}, c_{1}, c_{2}, c_{3}^{*}, c_{4}^{*}, \bar{c}_{3}^{*}, c_{5}, c_{6}, c_{7}\right)}{\partial\left(\delta_{1}, \delta_{2}, \delta_{3}, \delta_{4}, \delta_{5}, \delta_{6}, \delta_{7}, \delta_{8}, \ldots, \delta_{m}\right)}=10$

then (1) can have 18 limit cycles near $L_{0}$ for some $(\epsilon, \delta)$ near $\left(0, \delta_{0}\right)$.

Proof. (1) Because of the similarity in the proof, we only prove the conclusion for $l=9$ and omit the rest. By our assumptions, there exists $\delta_{0} \in \mathbb{R}^{m}$ such that $\bar{c}_{0}\left(\delta_{0}\right)=$ $\bar{c}_{3}^{*}\left(\delta_{0}\right)=c_{3}^{*}\left(\delta_{0}\right)=c_{4}^{*}\left(\delta_{0}\right)=0, c_{j}\left(\delta_{0}\right)=0, j=$ $0,1,2,5,6,7,8, c_{9}\left(\delta_{0}\right) \neq 0$, and

$$
\operatorname{rank} \frac{\partial\left(c_{0}, \bar{c}_{0}, c_{1}, c_{2}, c_{3}^{*}, c_{4}^{*}, \bar{c}_{3}^{*}, c_{5}, \ldots, c_{8}\right)}{\partial\left(\delta_{1}, \delta_{2}, \delta_{3}, \delta_{4}, \delta_{5}, \delta_{6}, \delta_{7}, \delta_{8}, \ldots, \delta_{m}\right)}=11 .
$$


By the implicit function theorem, we can take $\bar{c}_{0}, \bar{c}_{3}^{*}, c_{3}^{*}$, $c_{4}^{*}, c_{j}, j=0,1,2,5,6,7,8$ as free parameters varying near zero. Obviously, for these parameters varying near zero we have $\left|c_{9}\right| \geq\left|(1 / 2) c_{9}\left(\delta_{0}\right)\right|>0$. In the following we proceed the process by 9 steps.

Step 1. Fix $\left(c_{0}, \bar{c}_{0}, c_{1}, c_{2}, c_{3}^{*}, c_{4}^{*}, \bar{c}_{3}^{*}, c_{5}, c_{6}, c_{7}\right)=(0,0,0,0,0,0,0$, $0,0,0)$ and vary $c_{8}$ near 0 .

First, for $c_{8}=0$, we have by (26) $c_{9} M_{1}>0$ for $0<-h \ll 1$.

Let $0<\left|c_{8}\right| \ll 1$. Then $c_{9} M_{1}<0$ for $0<-h \ll 1$ if $c_{8} c_{9}>0$. Thus, $M_{1}$ has a zero. Hence, for $0<\left|c_{8}\right| \ll 1$,

(1) the condition $c_{8} c_{9}>0$ implies a distribution $(1,0)+0$ of one limit cycle.

Step 2. Fix $\left(c_{0}, \bar{c}_{0}, c_{1}, c_{2}, c_{3}^{*}, c_{4}^{*}, \bar{c}_{3}^{*}, c_{5}, c_{6}\right)=(0,0,0,0,0,0,0$, $0,0), c_{8} c_{9}>0$ and vary $c_{7}$ near 0 .

First, for $c_{7}=0$, we have by (26), (27), and (28) $c_{8} M_{1}<$ $0, c_{9} M_{2}<0$ for $0<-h \ll 1$, and $c_{8} M_{3}<0$ for $0<h \ll 1$.

Let $0<\left|c_{7}\right| \ll\left|c_{8}\right|$. Then $c_{8} M_{1}>0, c_{9} M_{2}>0$ for $0<$ $-h \ll 1$, and $c_{8} M_{3}>0$ for $0<h \ll 1$ if $c_{7} c_{8}>0, c_{7} c_{9}>0$. Thus, $M_{1}, M_{2}$, and $M_{3}$ each gets a zero and the zero of $M_{1}$ got in Step 1 still exists. Hence, for $0<\left|c_{7}\right| \ll\left|c_{8}\right| \ll 1$,

(2) the conditions $c_{7} c_{8}>0, c_{8} c_{9}>0$ imply a distribution $(2,1)+1$ of 4 limit cycles.

Step 3. Fix $\left(c_{0}, \bar{c}_{0}, c_{1}, c_{2}, c_{3}^{*}, c_{4}^{*}, \bar{c}_{3}^{*}, c_{5}\right)=(0,0,0,0,0,0,0,0)$, $c_{7} \neq 0$ and vary $c_{6}$ near 0 .

First, for $c_{6}=0$, we have by (26), (27), and (28) $c_{7} M_{1}>$ $0, c_{7} M_{2}>0$ for $0<-h \ll 1$, and $c_{7} M_{3}>0$ for $0<h \ll 1$.

Let $0<\left|c_{6}\right| \ll\left|c_{7}\right|$. Then $c_{7} M_{1}<0, c_{7} M_{2}<0$ for $0<$ $-h \ll 1$, and $c_{7} M_{3}>0$ for $0<h \ll 1$ if $c_{6} c_{7}<0$. Thus, $M_{1}$ and $M_{2}$ each gets a new zero and the zeros got in above steps still exist. Hence, for $0<\left|c_{6}\right| \ll\left|c_{7}\right| \ll\left|c_{8}\right| \ll 1$,

(3) the conditions $c_{6} c_{7}<0, c_{7} c_{8}>0, c_{8} c_{9}>0$ imply a distribution $(3,2)+1$ of 6 limit cycles.

Step 4. Fix $\left(c_{0}, \bar{c}_{0}, c_{1}, c_{2}, c_{3}^{*}, c_{4}^{*}, \bar{c}_{3}^{*}\right)=(0,0,0,0,0,0,0), c_{6} \neq 0$ and vary $c_{5}$ near 0 .

First, for $c_{5}=0$, we have by (26) and (28) $c_{6} M_{1}>0$ for $0<-h \ll 1$, and $c_{6} M_{3}<0$ for $0<h \ll 1$.

Let $0<\left|c_{5}\right| \ll\left|c_{6}\right|$. Then $c_{6} M_{1}<0$ for $0<-h \ll 1$, and $c_{6} M_{3}>0$ for $0<h \ll 1$ if $c_{5} c_{6}<0$. Thus, $M_{1}$ and $M_{3}$ each has a new zero and the zeros got in above steps still exist. Hence, for $0<\left|c_{5}\right| \ll\left|c_{6}\right| \ll\left|c_{7}\right| \ll\left|c_{8}\right| \ll 1$,

(4) the conditions $c_{5} c_{6}<0, c_{6} c_{7}<0, c_{7} c_{8}>0, c_{8} c_{9}>0$ imply a distribution $(4,2)+2$ of 8 limit cycles.

Step 5. Fix $\left(c_{0}, \bar{c}_{0}, c_{1}, c_{2}, c_{3}^{*}\right)=(0,0,0,0,0), c_{5} c_{6}<0$ and vary $\left(\bar{c}_{3}^{*}, c_{4}^{*}\right)$ near $(0,0)$ with $\widetilde{c}_{2}^{*}\left(=\bar{c}_{3}^{*}+c_{4}^{*}\right) \neq 0$.

First, for $\left(\bar{c}_{3}^{*}, c_{4}^{*}\right)=(0,0)$, we have by (26), (27), and (28) $c_{5} M_{1}>0, c_{6} M_{2}>0$ for $0<-h \ll 1$, and $c_{5} M_{3}<0$ for $0<h \ll 1$.

Let $0<\left|\bar{c}_{3}^{*}, c_{4}^{*}\right| \ll\left|c_{5}\right|$. Then

$$
\begin{gathered}
c_{5} M_{1}<0, \quad c_{6} M_{2}<0 \text { for } 0<-h \ll 1, \\
c_{5} M_{3}>0 \quad \text { for } 0<h \ll 1
\end{gathered}
$$

if $c_{4}^{*} c_{5}>0, \bar{c}_{3}^{*} c_{6}>0, \widetilde{c}_{2}^{*} c_{5}>0$, and

$$
\begin{gathered}
c_{5} M_{1}<0, \quad c_{6} M_{2}<0 \quad \text { for } 0<-h \ll 1, \\
c_{5} M_{3}<0 \quad \text { for } 0<h \ll 1
\end{gathered}
$$

if $c_{4}^{*} c_{5}>0, \bar{c}_{3}^{*} c_{6}>0, \widetilde{c}_{2}^{*} c_{5}<0$. Thus, $M_{1}, M_{2}$, and $M_{3}$ each has one more zero in the first case and $M_{1}$ and $M_{2}$ each has a new zero in the second case. And the zeros got in above steps still exist. Hence, for $0<\left|\bar{c}_{3}^{*}, c_{4}^{*}\right| \ll\left|c_{5}\right| \ll\left|c_{6}\right| \ll\left|c_{7}\right| \ll$ $\left|c_{8}\right| \ll 1$,

(5i) the conditions $\bar{c}_{3}^{*} c_{4}^{*}<0, \bar{c}_{3}^{*} \widetilde{c}_{2}^{*}<0, c_{4}^{*} c_{5}>0, c_{5} c_{6}<$ $0, c_{6} c_{7}<0, c_{7} c_{8}>0, c_{8} c_{9}>0$ imply a distribution $(5,3)+3$ of 11 limit cycles, and

(5ii) the conditions $\bar{c}_{3}^{*} c_{4}^{*}<0, \bar{c}_{3}^{*} \widetilde{c}_{2}^{*}>0, c_{4}^{*} c_{5}>0, c_{5} c_{6}<$ $0, c_{6} c_{7}<0, c_{7} c_{8}>0, c_{8} c_{9}>0$ imply a distribution $(5,3)+2$ of 10 limit cycles.

Step 6. Fix $\left(c_{0}, \bar{c}_{0}, c_{1}, c_{2}\right)=(0,0,0,0), c_{4}^{*} \bar{c}_{3}^{*}<0$ with $\widetilde{c}_{2}^{*} \neq 0$ and vary $c_{3}^{*}$ near 0 .

First, for $c_{3}^{*}=0$, we have by (26) and (27) $c_{4}^{*} M_{1}<0$ and $\bar{c}_{3}^{*} M_{2}<0$ for $0<-h \ll 1$.

Let $0<\left|c_{3}^{*}\right| \ll\left|c_{4}^{*}, \bar{c}_{3}^{*}\right|$. Then $c_{4}^{*} M_{1}>0$ and $\bar{c}_{3}^{*} M_{2}>0$ for $0<-h \ll 1$, if $c_{3}^{*} c_{4}^{*}<0, \bar{c}_{3}^{*} c_{3}^{*}>0$. Thus, $M_{1}$ and $M_{2}$ each gets a new zero and the zeros got in above steps still exist. Hence, for $0<\left|c_{3}^{*}\right| \ll\left|c_{4}^{*}, \bar{c}_{3}^{*}\right| \ll\left|c_{5}\right| \ll\left|c_{6}\right| \ll\left|c_{7}\right| \ll\left|c_{8}\right| \ll 1$,

(6i) the conditions $c_{3}^{*} c_{4}^{*}<0, \bar{c}_{3}^{*} c_{4}^{*}<0, \bar{c}_{3}^{*} \widetilde{c}_{2}^{*}<0, c_{4}^{*} c_{5}>$ $0, c_{5} c_{6}<0, c_{6} c_{7}<0, c_{7} c_{8}>0, c_{8} c_{9}>0$ imply a distribution $(6,4)+3$ of 13 limit cycles,

(6ii) the conditions $c_{3}^{*} c_{4}^{*}<0, \bar{c}_{3}^{*} c_{4}^{*}<0, \bar{c}_{3}^{*} \widetilde{c}_{2}^{*}>0, c_{4}^{*} c_{5}>$ $0, c_{5} c_{6}<0, c_{6} c_{7}<0, c_{7} c_{8}>0, c_{8} c_{9}>0$ imply a distribution $(6,4)+2$ of 12 limit cycles.

Step 7. Fix $\left(c_{0}, \bar{c}_{0}, c_{1}\right)=(0,0,0), c_{3}^{*} \widetilde{c}_{2}^{*} \neq 0$ and vary $c_{2}$ near 0 .

First, for $c_{2}=0$, we have by (26) and (28) $c_{3}^{*} M_{1}<0$ for $0<-h \ll 1$ and $\widetilde{c}_{2}^{*} M_{3}>0$ for $0<h \ll 1$.

Let $0<\left|c_{2}\right| \ll\left|c_{3}^{*}\right| \ll\left|c_{4}^{*}, \bar{c}_{3}^{*}\right|$. Then

$$
\begin{array}{cc}
c_{3}^{*} M_{1}>0 & \text { for } 0<-h \ll 1, \\
\widetilde{c}_{2}^{*} M_{3}<0 & \text { for } 0<h \ll 1
\end{array}
$$

if $c_{2} c_{3}^{*}>0, \widetilde{c}_{2}^{*} c_{2}>0$, and

$$
\begin{array}{cc}
c_{3}^{*} M_{1}>0 & \text { for } 0<-h \ll 1, \\
\widetilde{c}_{2}^{*} M_{3}>0 & \text { for } 0<h \ll 1
\end{array}
$$

if $c_{2} c_{3}^{*}>0, \widetilde{c}_{2}^{*} c_{2}<0$. Thus, $M_{1}$ and $M_{3}$ each gets one more zero in the first case and only $M_{1}$ has a new zero in the second case. And the zeros got in above steps still exist. Hence, for $0<\left|c_{2}\right| \ll\left|c_{3}^{*}\right| \ll\left|c_{4}^{*}, \bar{c}_{3}^{*}\right| \ll\left|c_{5}\right| \ll\left|c_{6}\right| \ll\left|c_{7}\right| \ll\left|c_{8}\right| \ll 1$,

(7i) the conditions $c_{2} c_{3}^{*}>0, c_{3}^{*} c_{4}^{*}<0, \bar{c}_{3}^{*} c_{4}^{*}<0, \bar{c}_{3}^{*} \widetilde{c}_{2}^{*}>$ $0, c_{4}^{*} c_{5}>0, c_{5} c_{6}<0, c_{6} c_{7}<0, c_{7} c_{8}>0, c_{8} c_{9}>0$ imply a distribution $(7,4)+3$ of 14 limit cycles, 
(7ii) the conditions $c_{2} c_{3}^{*}>0, c_{3}^{*} c_{4}^{*}<0, \bar{c}_{3}^{*} c_{4}^{*}<0, \bar{c}_{3}^{*} \widetilde{c}_{2}^{*}<$ $0, c_{4}^{*} c_{5}>0, c_{5} c_{6}<0, c_{6} c_{7}<0, c_{7} c_{8}>0, c_{8} c_{9}>0$ imply a distribution $(7,4)+3$ of 14 limit cycles.

Step 8. Fix $\left(c_{0}, \bar{c}_{0}\right)=(0,0), c_{2} c_{3}^{*}>0$ and vary $c_{1}$ near 0 .

First, for $c_{1}=0$, we have by (26), (27), and (28) $c_{2} M_{1}>$ $0, c_{3}^{*} M_{2}>0$ for $0<-h \ll 1$, and $c_{2} M_{3}<0$ for $0<h \ll 1$.

Let $0<\left|c_{1}\right| \ll\left|c_{2}\right|$. Then $c_{2} M_{1}<0, c_{3}^{*} M_{2}<0$ for $0<$ $-h \ll 1$, and $c_{2} M_{3}>0$ for $0<h \ll 1$ if $c_{1} c_{2}<0$. Thus, $M_{1}, M_{2}$, and $M_{3}$ each gets a new zero and the zeros got in above steps still exist. Hence, for $0<\left|c_{1}\right| \ll\left|c_{2}\right| \ll\left|c_{3}^{*}\right| \ll$ $\left|c_{4}^{*}, \bar{c}_{3}^{*}\right| \ll\left|c_{5}\right| \ll\left|c_{6}\right| \ll\left|c_{7}\right| \ll\left|c_{8}\right| \ll 1$,

(8) the conditions $c_{1} c_{2}<0, c_{2} c_{3}^{*}>0, c_{3}^{*} c_{4}^{*}<0, \bar{c}_{3}^{*} c_{4}^{*}<$ $0, \bar{c}_{3}^{*} \widetilde{c}_{2}^{*}>0$ (or $\left.\bar{c}_{3}^{*} \widetilde{c}_{2}^{*}<0\right), c_{4}^{*} c_{5}>0, c_{5} c_{6}<0, c_{6} c_{7}<$ $0, c_{7} c_{8}>0, c_{8} c_{9}>0$ imply a distribution $(8,5)+4$ of 17 limit cycles.

Step 9. Fix $c_{1} \neq 0$ and vary $\left(c_{0}, \bar{c}_{0}\right)$ near $(0,0)$.

First, for $\left(c_{0}, \bar{c}_{0}\right)=(0,0)$, we have by (26), (27), and (28) $c_{1} M_{1}>0, c_{1} M_{2}>0$, for $0<-h \ll 1$, and $c_{1} M_{3}<0$ for $0<h \ll 1$.

Let $0<\left|c_{0}, \bar{c}_{0}\right| \ll\left|c_{1}\right|$. Then

$$
\begin{gathered}
c_{1} M_{1}<0, \quad c_{1} M_{2}<0, \quad \text { for } 0<-h \ll 1, \\
c_{1} M_{3}<0 \quad \text { for } 0<h \ll 1
\end{gathered}
$$

if $c_{0} c_{1}<0, \bar{c}_{0} c_{1}<0$,

$$
\begin{gathered}
c_{1} M_{1}<0, \quad c_{1} M_{2}>0, \quad \text { for } 0<-h \ll 1, \\
c_{1} M_{3}>0 \text { for } 0<h \ll 1
\end{gathered}
$$

if $c_{0} c_{1}<0, \bar{c}_{0} c_{1}>0$ and $\left(c_{0}+\bar{c}_{0}\right) c_{1}>0$, and

$$
\begin{gathered}
c_{1} M_{1}>0, \quad c_{1} M_{2}<0, \quad \text { for } 0<-h \ll 1, \\
c_{1} M_{3}>0 \quad \text { for } 0<h \ll 1
\end{gathered}
$$

if $c_{0} c_{1}>0, \bar{c}_{0} c_{1}<0$ and $\left(c_{0}+\bar{c}_{0}\right) c_{1}>0$. Thus, we have correspondingly (a) $M_{1}$ and $M_{2}$ each has a new zero, (b) $M_{1}$ and $M_{3}$ each has a new zero, or (c) $M_{2}$ and $M_{3}$ each has a new zero. And the zeros got in above steps still exist.

Hence, for $0<\left|c_{0}, \bar{c}_{0}\right| \ll\left|c_{1}\right| \ll\left|c_{2}\right| \ll\left|c_{3}^{*}\right| \ll\left|c_{4}^{*}, \bar{c}_{3}^{*}\right| \ll$ $\left|c_{5}\right| \ll\left|c_{6}\right| \ll\left|c_{7}\right| \ll\left|c_{8}\right| \ll 1$,

(9i) the conditions $c_{0} \bar{c}_{0}>0, c_{0} c_{1}<0, c_{1} c_{2}<0, c_{2} c_{3}^{*}>$ $0, c_{3}^{*} c_{4}^{*}<0, \bar{c}_{3}^{*} c_{4}^{*}<0, \bar{c}_{3}^{*} \widetilde{c}_{2}^{*}>0$ (or $\bar{c}_{3}^{*} \widetilde{c}_{2}^{*}<$ $0), c_{4}^{*} c_{5}>0, c_{5} c_{6}<0, c_{6} c_{7}<0, c_{7} c_{8}>0, c_{8} c_{9}>0$ imply a distribution $(9,6)+4$ of 19 limit cycles,

(9ii) the conditions $c_{0} \bar{c}_{0}<0, c_{0} c_{1}<0,\left(c_{0}+\bar{c}_{0}\right) c_{1}>$ $0, c_{1} c_{2}<0, c_{2} c_{3}^{*}>0, c_{3}^{*} c_{4}^{*}<0, \bar{c}_{3}^{*} c_{4}^{*}<0, \bar{c}_{3}^{*} \widetilde{c}_{2}^{*}>$ 0 (or $\left.\bar{c}_{3}^{*} \widetilde{c}_{2}^{*}<0\right), c_{4}^{*} c_{5}>0, c_{5} c_{6}<0, c_{6} c_{7}<0, c_{7} c_{8}>$ $0, c_{8} c_{9}>0$ imply a distribution $(9,5)+5$ of 19 limit cycles,

(9iii) the conditions $c_{0} \bar{c}_{0}<0, c_{0} c_{1}>0,\left(c_{0}+\bar{c}_{0}\right) c_{1}>$ $0, c_{1} c_{2}<0, c_{2} c_{3}^{*}>0, c_{3}^{*} c_{4}^{*}<0, \bar{c}_{3}^{*} c_{4}^{*}<0, \bar{c}_{3}^{*} \widetilde{c}_{2}^{*}>$ 0 (or $\left.\bar{c}_{3}^{*} \widetilde{c}_{2}^{*}<0\right), c_{4}^{*} c_{5}>0, c_{5} c_{6}<0, c_{6} c_{7}<0, c_{7} c_{8}>$ $0, c_{8} c_{9}>0$ imply a distribution $(8,6)+5$ of 19 limit cycles.

Thus we get the conclusion for $l=9$.
(2) By our assumptions in case (2) and the implicit function theorem we can take $c_{0}, \bar{c}_{0}, c_{1}, c_{2}, c_{3}^{*}, c_{4}^{*}, \bar{c}_{3}^{*}$ as free parameters varying near zero. Obviously, for these parameters varying near zero we have $c_{5} c_{6}<0$. By a similar argument in the above proof, we can prove that for $0<$ $\left|c_{0}, \bar{c}_{0}\right| \ll\left|c_{1}\right| \ll\left|c_{2}\right| \ll\left|c_{3}^{*}\right| \ll\left|c_{4}^{*}, \bar{c}_{3}^{*}\right| \ll 1$,

(i) the conditions $c_{0} \bar{c}_{0}>0, c_{0} c_{1}<0, c_{1} c_{2}<0, c_{2} c_{3}^{*}>$ $0, c_{3}^{*} c_{4}^{*}<0, \bar{c}_{3}^{*} c_{4}^{*}<0, \bar{c}_{3}^{*} \widetilde{c}_{2}^{*}>0$ (or $\bar{c}_{3}^{*} \widetilde{c}_{2}^{*}<$ $0), c_{4}^{*} c_{5}>0$ imply a distribution $(5,4)+2$ of 11 limit cycles,

(ii) the conditions $c_{0} \bar{c}_{0}<0, c_{0} c_{1}<0,\left(c_{0}+\bar{c}_{0}\right) c_{1}>$ $0, c_{1} c_{2}<0, c_{2} c_{3}^{*}>0, c_{3}^{*} c_{4}^{*}<0, \bar{c}_{3}^{*} c_{4}^{*}<0, \bar{c}_{3}^{*} \bar{c}_{2}^{*}>$ 0 (or $\left.\bar{c}_{3}^{*} \widetilde{c}_{2}^{*}<0\right), c_{4}^{*} c_{5}>0$ imply a distribution $(5,3)+3$ of 11 limit cycles,

(iii) the conditions $c_{0} \bar{c}_{0}<0, c_{0} c_{1}>0,\left(c_{0}+\bar{c}_{0}\right) c_{1}>$ $0, c_{1} c_{2}<0, c_{2} c_{3}^{*}>0, c_{3}^{*} c_{4}^{*}<0, \bar{c}_{3}^{*} c_{4}^{*}<0, \bar{c}_{3}^{*} \widetilde{c}_{2}^{*}>$ 0 (or $\left.\bar{c}_{3}^{*} \widetilde{c}_{2}^{*}<0\right), c_{4}^{*} c_{5}>0$ imply a distribution $(4,4)+3$ of 11 limit cycles.

(3) By our assumptions in case (3) and the implicit function theorem we can take $c_{0}, \bar{c}_{0}, c_{1}$, $c_{2}, c_{3}^{*}, c_{4}^{*}, \bar{c}_{3}^{*}, c_{5}, c_{6}$, and $c_{7}$ as free parameters varying near zero. Obviously, for these parameters varying near zero we have $c_{8} c_{9}>0$. By a similar argument used in proving case (1), we can prove that for $0<\left|c_{0}, \bar{c}_{0}\right| \ll\left|c_{1}\right| \ll\left|c_{2}\right| \ll\left|c_{3}^{*}\right| \ll$ $\left|c_{4}^{*}, \bar{c}_{3}^{*}\right| \ll\left|c_{5}\right| \ll\left|c_{6}\right| \ll\left|c_{7}\right| \ll 1$,

(i) the conditions $c_{0} \bar{c}_{0}>0, c_{0} c_{1}<0, c_{1} c_{2}<0, c_{2} c_{3}^{*}>$ $0, c_{3}^{*} c_{4}^{*}<0, \bar{c}_{3}^{*} c_{4}^{*}<0, \bar{c}_{3}^{*} \widetilde{c}_{2}^{*}>0$ (or $\bar{c}_{3}^{*} \widetilde{c}_{2}^{*}<$ $0), c_{4}^{*} c_{5}>0, c_{5} c_{6}<0, c_{6} c_{7}<0, c_{7} c_{8}>0$ imply a distribution $(8,6)+4$ of 18 limit cycles,

(ii) the conditions $c_{0} \bar{c}_{0}<0, c_{0} c_{1}<0,\left(c_{0}+\bar{c}_{0}\right) c_{1}>$ $0, c_{1} c_{2}<0, c_{2} c_{3}^{*}>0, c_{3}^{*} c_{4}^{*}<0, \bar{c}_{3}^{*} c_{4}^{*}<0, \bar{c}_{3}^{*} \widetilde{c}_{2}^{*}>$ $0\left(\right.$ or $\left.\bar{c}_{3}^{*} \widetilde{c}_{2}^{*}<0\right), c_{4}^{*} c_{5}>0, c_{5} c_{6}<0, c_{6} c_{7}<0, c_{7} c_{8}>$ 0 imply a distribution $(8,5)+5$ of 18 limit cycles,

(iii) the conditions $c_{0} \bar{c}_{0}<0, c_{0} c_{1}>0,\left(c_{0}+\bar{c}_{0}\right) c_{1}>$ $0, c_{1} c_{2}<0, c_{2} c_{3}^{*}>0, c_{3}^{*} c_{4}^{*}<0, \bar{c}_{3}^{*} c_{4}^{*}<0, \bar{c}_{3}^{*} \widetilde{c}_{2}^{*}>$ 0 (or $\left.\bar{c}_{3}^{*} \widetilde{c}_{2}^{*}<0\right), c_{4}^{*} c_{5}>0, c_{5} c_{6}<0, c_{6} c_{7}<0, c_{7} c_{8}>$ 0 imply a distribution $(7,6)+5$ of 18 limit cycles.

This completes the proof.

\section{An Application}

Consider a Liénard system of the form

$$
\dot{x}=y, \quad \dot{y}=-(x+1)^{2} x^{3}\left(x^{2}-\frac{1}{4} x-\frac{1}{2}\right)-\epsilon f(x, \delta) y,
$$

where

$$
\begin{array}{r}
f(x, \delta)=\sum_{j=0}^{n} a_{j} x^{j}, \quad \delta=\left(a_{0}, a_{1}, \ldots, a_{n}\right), \\
8 \leq n \leq 12 .
\end{array}
$$

System (54) $\left.\right|_{\epsilon=0}$ is Hamiltonian with

$$
H(x, y)=\frac{1}{2} y^{2}+\frac{1}{8}(x+1)^{3} x^{4}(x-1) .
$$


We have the following theorem.

Theorem 5. Let $C(n)$ denote the maximal number of limit cycles of the system (54) for $\epsilon$ small and all $\delta$. Then we have $C(11) \geq 18, C(n) \geq 2 n-5(n=8,9,10,12)$.

Proof. It is easy to verify that the unperturbed system has a compound loop $L_{0}=L_{1} \cup L_{2} \cup L_{3} \cup\left\{S_{1}, S_{2}\right\}$ with a cusp $S_{1}(-1,0)$ of order one and a nilpotent saddle $S_{2}(0,0)$ of order one, $L_{1}, L_{2}$ are heteroclinic orbits satisfying $\omega\left(L_{1}\right)=\alpha\left(L_{2}\right)=$ $S_{2}$ and $\omega\left(L_{2}\right)=\alpha\left(L_{1}\right)=S_{1}$, and $L_{3}$ is a homoclinic loop to $S_{2}$. Inside $L^{*}=L_{1}+L_{2}\left(L_{3}\right.$, resp. $)$, there is a center $C_{1}((1 / 8)(1-$ $\sqrt{33}), 0)\left(C_{2}((1 / 8)(1+\sqrt{33}), 0)\right.$, resp.).

Because of the similarity in the proof, here we only prove the case for $n=11$ and omit the rest of the proof.

Let $n=11$. By Theorem 3, we obtain

$$
\begin{aligned}
& c_{0}(\delta)=M_{1}(0, \delta)=-\oint_{L^{*}} f(x, \delta) y d x=-\sum_{j=0}^{11} a_{j} I_{1}^{j}, \\
& \bar{c}_{0}(\delta)=M_{2}(0, \delta)=-\oint_{L_{3}} f(x, \delta) y d x=-\sum_{j=0}^{11} a_{j} I_{2}^{j},
\end{aligned}
$$

where

$$
\begin{aligned}
I_{1}^{j} & =\oint_{L^{*}} x^{j} y d x=2 \int_{-1}^{0} x^{j} y d x \\
& =\int_{-1}^{0} x^{j+2}(x+1) \sqrt{1-x^{2}} d x, \quad j=0,1, \ldots, 11, \\
I_{2}^{j} & =\oint_{L_{3}} x^{j} y d x=2 \int_{0}^{1} x^{j} y d x \\
& =\int_{0}^{1} x^{j+2}(x+1) \sqrt{1-x^{2}} d x, \quad j=0,1, \ldots, 11 .
\end{aligned}
$$

Therefore,

$$
\begin{aligned}
c_{0}(\delta)= & -a_{0}\left(\frac{\pi}{16}-\frac{2}{15}\right)-a_{1}\left(\frac{\pi}{32}-\frac{2}{15}\right)-a_{2}\left(\frac{\pi}{32}-\frac{8}{105}\right) \\
& -a_{3}\left(\frac{5 \pi}{256}-\frac{8}{105}\right)-a_{4}\left(\frac{5 \pi}{256}-\frac{16}{315}\right) \\
& -a_{5}\left(\frac{7 \pi}{512}-\frac{16}{315}\right)-a_{6}\left(\frac{7 \pi}{512}-\frac{128}{3465}\right) \\
& -a_{7}\left(\frac{21 \pi}{2048}-\frac{128}{3465}\right)-a_{8}\left(\frac{21 \pi}{2048}-\frac{256}{9009}\right) \\
& -a_{9}\left(\frac{33 \pi}{4096}-\frac{256}{9009}\right)-a_{10}\left(\frac{33 \pi}{4096}-\frac{1024}{45045}\right) \\
& -a_{11}\left(\frac{429 \pi}{65536}-\frac{1024}{45045}\right),
\end{aligned}
$$

$$
\begin{aligned}
\bar{c}_{0}(\delta)= & -a_{0}\left(\frac{\pi}{16}+\frac{2}{15}\right)-a_{1}\left(\frac{\pi}{32}+\frac{2}{15}\right)-a_{2}\left(\frac{\pi}{32}+\frac{8}{105}\right) \\
& -a_{3}\left(\frac{5 \pi}{256}+\frac{8}{105}\right)-a_{4}\left(\frac{5 \pi}{256}+\frac{16}{315}\right) \\
& -a_{5}\left(\frac{7 \pi}{512}+\frac{16}{315}\right)-a_{6}\left(\frac{7 \pi}{512}+\frac{128}{3465}\right) \\
& -a_{7}\left(\frac{21 \pi}{2048}+\frac{128}{3465}\right)-a_{8}\left(\frac{21 \pi}{2048}+\frac{256}{9009}\right) \\
& -a_{9}\left(\frac{33 \pi}{4096}+\frac{256}{9009}\right)-a_{10}\left(\frac{33 \pi}{4096}+\frac{1024}{45045}\right) \\
& -a_{11}\left(\frac{429 \pi}{65536}+\frac{1024}{45045}\right) .
\end{aligned}
$$

Note that $S_{2}$ is a nilpotent saddle of order one and $\bar{h}_{4}=-1 / 8$. By (23), we have

$$
\begin{aligned}
c_{1}(\delta) & =c_{1}\left(S_{2}, \delta\right)=2^{9 / 4}\left|\widetilde{A}_{0}\right| a_{0}, \\
c_{3}(\delta) & =c_{2}\left(S_{2}, \delta\right)=a_{1}+O_{1}\left(a_{0}\right), \\
c_{6}(\delta) & =c_{4}\left(S_{2}, \delta\right)=2^{3 / 4}\left|\widetilde{A}_{2}\right|\left(-21 a_{0}+12 a_{1}-8 a_{2}\right), \\
c_{7}(\delta) & =c_{5}\left(S_{2}, \delta\right) \\
& =2^{1 / 4} \frac{\left|\widetilde{A_{0}}\right|}{7}\left(\frac{2035}{4} a_{0}-310 a_{1}+180 a_{2}-80 a_{3}+32 a_{4}\right), \\
c_{9}(\delta) & =c_{6}\left(S_{2}, \delta\right) \\
& =\frac{333}{4} a_{0}-\frac{207}{4} a_{1}+29 a_{2}-15 a_{3}+6 a_{4}-2 a_{5} .
\end{aligned}
$$

Making the transformation $x=u-1, y=v$, system (54) becomes

$$
\dot{u}=v, \quad \dot{v}=-u^{2}(u-1)^{3}\left(u^{2}-\frac{9}{4} u+\frac{3}{4}\right)-\epsilon \widetilde{f}(u, \delta) v .
$$

Then we have

$$
\begin{aligned}
& H(u, v)=\frac{v^{2}}{2}+ \frac{1}{8}\left(u^{8}-6 u^{7}+14 u^{6}-16 u^{5}+9 u^{4}-2 u^{3}\right) \\
& \tilde{h}_{3}=-\frac{1}{4} \\
& \tilde{f}(u, \delta)=\sum_{j=0}^{11} a_{j}(u-1)^{j}=\sum_{j=0}^{11}(-1)^{j} a_{j} \\
&+\sum_{j=1}^{11}(-1)^{(j-1)} j a_{j} u+\sum_{j=2}^{11}(-1)^{(j-2)} C_{j}^{2} a_{j} u^{2} \\
&+\sum_{j=3}^{11}(-1)^{(j-3)} C_{j}^{3} a_{j} u^{3}+\sum_{j=4}^{11}(-1)^{(j-4)} C_{j}^{4} a_{j} u^{4}
\end{aligned}
$$




$$
\begin{aligned}
& +\sum_{j=5}^{11}(-1)^{(j-5)} C_{j}^{5} a_{j} u^{5}+\sum_{j=6}^{11}(-1)^{(j-6)} C_{j}^{6} a_{j} u^{6} \\
& +\sum_{j=7}^{11}(-1)^{(j-7)} C_{j}^{7} a_{j} u^{7}+\sum_{j=8}^{11}(-1)^{(j-8)} C_{j}^{8} a_{j} u^{8} \\
& +\sum_{j=9}^{11}(-1)^{(j-9)} C_{j}^{9} a_{j} u^{9}+\left(a_{10}-11 a_{11}\right) u^{10}+a_{11} u^{11} .
\end{aligned}
$$

By (19), we have

$$
\begin{gathered}
c_{2}(\delta)=c_{1}\left(S_{1}, \delta\right)=2^{13 / 6} \sum_{j=0}^{11}(-1)^{j} a_{j}, \\
c_{5}(\delta)=c_{3}\left(S_{1}, \delta\right)=2^{17 / 6}\left[\sum_{j=1}^{11}(-1)^{j}(j-3) a_{j}-3 a_{0}\right], \\
c_{8}(\delta)=c_{4}\left(S_{1}, \delta\right) \\
=2^{13 / 6}\left(-\frac{1316}{3} a_{0}+272 a_{1}-\frac{460}{3} a_{2}+\frac{224}{3} a_{3}\right. \\
\quad-28 a_{4}+\frac{16}{3} a_{5}+\frac{4}{3} a_{6}-\frac{4}{3} a_{8} \\
\left.\quad-\frac{16}{3} a_{9}+28 a_{10}-\frac{224}{3} a_{11}\right) .
\end{gathered}
$$

Note that

$$
\begin{aligned}
& c_{1}(\delta)=c_{1}\left(S_{2}, \delta\right)=2^{9 / 4}\left|\widetilde{A}_{0}\right| a_{0}, \\
& c_{2}(\delta)=c_{1}\left(S_{1}, \delta\right)=2^{13 / 6} \sum_{j=0}^{11}(-1)^{j} a_{j}, \\
& c_{3}(\delta)=c_{2}\left(S_{2}, \delta\right)=a_{1}+O_{1}\left(a_{0}\right) .
\end{aligned}
$$

We have $c_{1}(\delta)=c_{2}(\delta)=c_{3}(\delta)=0$ if and only if $a_{0}=a_{1}=0$, and $a_{11}=\sum_{i=2}^{10}(-1)^{i} a_{i}$. It implies further that

$$
\begin{aligned}
c_{4}(\delta) & =\oint_{L^{*}}\left(p_{x}+q_{y}\right) d t=-\oint_{L^{*}} f(x, \delta) d t \\
& =-\oint_{L^{*}} \frac{f(x, \delta)}{y} d x=-2 \int_{-1}^{0} \frac{f(x, \delta)}{y} d x \\
& =-2 \int_{-1}^{0} \frac{1}{y}\left(\sum_{i=0}^{11} a_{i} x^{i}\right) d x \\
& =-4 \int_{-1}^{0} \frac{1}{x^{2}(x+1) \sqrt{1-x^{2}}} \\
& \times\left(\sum_{i=2}^{10} a_{i} x^{i}+\sum_{i=2}^{10}(-1)^{i} a_{i} x^{11}\right) d x \\
& =-4 \sum_{i=2}^{10} a_{i} \int_{-1}^{0} f_{i}(x) d x,
\end{aligned}
$$

where

$$
f_{i}(x)=\frac{x^{i-2}\left[1+(-1)^{i} x^{(11-i)}\right]}{(x+1) \sqrt{1-x^{2}}}, \quad i=2,3, \ldots, 10 .
$$

Similarly,

$$
\begin{aligned}
\bar{c}_{3}(\delta) & =\oint_{L_{3}}\left(p_{x}+q_{y}\right) d t=-\oint_{L_{3}} f(x, \delta) d t \\
& =-\oint_{L_{3}} \frac{f(x, \delta)}{y} d x=-2 \int_{0}^{1} \frac{f(x, \delta)}{y} d x \\
& =-4 \sum_{i=2}^{10} a_{i} \int_{0}^{1} f_{i}(x) d x .
\end{aligned}
$$

Therefore,

$$
\begin{aligned}
c_{4}(\delta)= & -4 a_{2}\left(\frac{315 \pi}{256}+\frac{93}{35}\right)+4 a_{3}\left(\frac{187 \pi}{256}+\frac{93}{35}\right) \\
& -4 a_{4}\left(\frac{187 \pi}{256}+\frac{58}{35}\right)+4 a_{5}\left(\frac{123 \pi}{256}+\frac{58}{35}\right) \\
& -4 a_{6}\left(\frac{123 \pi}{256}+\frac{104}{105}\right)+4 a_{7}\left(\frac{75 \pi}{256}+\frac{104}{105}\right) \\
& -4 a_{8}\left(\frac{75 \pi}{256}+\frac{16}{35}\right)+4 a_{9}\left(\frac{35 \pi}{256}+\frac{16}{35}\right) \\
& -4 a_{10}\left(\frac{35 \pi}{256}\right), \\
\bar{c}_{3}(\delta)= & -4 a_{2}\left(\frac{315 \pi}{256}-\frac{93}{35}\right)+4 a_{3}\left(\frac{187 \pi}{256}-\frac{93}{35}\right) \\
& -4 a_{4}\left(\frac{187 \pi}{256}-\frac{58}{35}\right)+4 a_{5}\left(\frac{123 \pi}{256}-\frac{58}{35}\right) \\
& -4 a_{6}\left(\frac{123 \pi}{256}-\frac{104}{105}\right)+4 a_{7}\left(\frac{75 \pi}{256}-\frac{104}{105}\right) \\
& -4 a_{8}\left(\frac{75 \pi}{256}-\frac{16}{35}\right)+4 a_{9}\left(\frac{35 \pi}{256}-\frac{16}{35}\right) \\
& -4 a_{10}\left(\frac{35 \pi}{256}\right) .
\end{aligned}
$$

Let $c_{4}^{*}(\delta)=\left.c_{4}(\delta)\right|_{c_{1}=c_{2}=c_{3}=0}, \bar{c}_{3}^{*}(\delta)=\bar{c}_{3}(\delta)_{c_{1}=c_{3}=0}$, $c_{3}^{*}(\delta)=\left.c_{3}(\delta)\right|_{c_{1}(\delta)=0}$. Furthermore, one sees that equations $c_{0}(\delta)=\bar{c}_{0}(\delta)=c_{1}(\delta)=c_{2}(\delta)=c_{3}^{*}(\delta)=c_{4}^{*}(\delta)=\bar{c}_{3}^{*}(\delta)=$ $c_{5}(\delta)=c_{6}(\delta)=c_{7}(\delta)=0$ have the solution $a_{0}=a_{1}=a_{2}=$ $0, a_{3}=(13 / 74) a_{9}-(1 / 2) a_{7}, a_{4}=(65 / 148) a_{9}-(5 / 4) a_{7}, a_{5}=$ $-(16 / 37) a_{9}, a_{6}=(7 / 4) a_{7}-(255 / 148) a_{9}, a_{8}=(96 / 37) a_{9}, a_{10}=$ $-(53 / 37) a_{9}, a_{11}=-(32 / 37) a_{9}$, which gives $c_{8}(\delta)=$ $(55 / 37) 2^{31 / 6} a_{9}, c_{9}(\delta)=(32 / 37) a_{9}$. And further, $c_{8}(\delta) c_{9}(\delta)>$ 0 if $a_{9} \neq 0$. Thus, fix $a_{9} \neq 0$ and take $\delta_{0}=\left(0,0,0,(13 / 74) a_{9}-\right.$ $(1 / 2) a_{7},(65 / 148) a_{9}-(5 / 4) a_{7},-(16 / 37) a_{9},(7 / 4) a_{7}$ $\left.(255 / 148) a_{9}, a_{7},(96 / 37) a_{9}, a_{9},-(53 / 37) a_{9},-(32 / 37) a_{9}\right)$.

Then we have

$$
\operatorname{rank} \frac{\partial\left(c_{0}, \bar{c}_{0}, c_{1}, c_{2}, c_{3}^{*}, c_{4}^{*}, \bar{c}_{3}^{*}, c_{5}, c_{6}, c_{7}\right)}{\partial\left(a_{0}, a_{1}, a_{2}, a_{3}, a_{4}, a_{5}, a_{6}, a_{7}, a_{8}, a_{9}, a_{10}, a_{11}\right)}=10
$$


Hence by Theorem 4(3), we know that there are 18 limit cycles near $L_{0}$ for some $\delta$ near $\delta_{0}$. This ends the proof.

\section{Conflict of Interests}

The authors declare that there is no conflict of interests regarding the publication of this paper.

\section{Acknowledgments}

The project was supported by National Natural Science Foundation of China (11271261) and FP7-PEOPLE-2012-IRSES316338 .

\section{References}

[1] M. Han, "Asymptotic expansions of Melnikov functions and limit cycle bifurcations," International Journal of Bifurcation and Chaos in Applied Sciences and Engineering, vol. 22, no. 12, Article ID 1250296, 30 pages, 2012.

[2] A. Atabaigi, H. R. Z. Zangeneh, and R. Kazemi, "Limit cycle bifurcation by perturbing a cuspidal loop of order 2 in a Hamiltonian system," Nonlinear Analysis: Theory, Methods \& Applications, vol. 75, no. 4, pp. 1945-1958, 2012.

[3] M. Han, H. Zang, and J. Yang, "Limit cycle bifurcations by perturbing a cuspidal loop in a Hamiltonian system," Journal of Differential Equations, vol. 246, no. 1, pp. 129-163, 2009.

[4] M. Han, J. Yang, and D. Xiao, "Limit cycle bifurcations near a double homoclinic loop with a nilpotent saddle," International Journal of Bifurcation and Chaos in Applied Sciences and Engineering, vol. 22, no. 8, Article ID 1250189, 33 pages, 2012.

[5] M. Han, Bifurcation Theory of Limit Cycles, Science Press, 2013.

[6] Z. Hu, B. Gao, and V. G. Romanovski, "Limit cycle bifurcations from centers of symmetric Hamiltonian systems pertrbed by cubic polynomials," International Journal of Bifurcation and Chaos, vol. 23, no. 3, Article ID 1350043, 20 pages, 2013.

[7] J. Jiang, "Bifurcation of limit cycles for a quartic nearHamiltonian system by perturbing a nilpotent center," Journal of Mathematical Analysis and Applications, vol. 365, no. 1, pp. 376-384, 2010.

[8] J. Li, T. Zhang, and M. Han, "Bifurcation of limit cycles from a heteroclinic loop with two cusps," Chaos, Solitons \& Fractals, vol. 62-63, pp. 44-54, 2014.

[9] X. Sun, M. Han, and J. Yang, "Bifurcation of limit cycles from a heteroclinic loop with a cusp," Nonlinear Analysis: Theory, Methods \& Applications, vol. 74, no. 9, pp. 2948-2965, 2011.

[10] J. Yang, "On the limit cycles of a kind of Liénard system with a nilpotent center under perturbations," The Journal of Applied Analysis and Computation, vol. 2, no. 3, pp. 325-339, 2012.

[11] H. Zang, M. Han, and D. Xiao, "On Melnikov functions of a homoclinic loop through a nilpotent saddle for planar nearHamiltonian systems," Journal of Differential Equations, vol. 245, no. 4, pp. 1086-1111, 2008.

[12] H. Zang, Z. Wang, and T. Zhang, "Bifurcations and distribution of limit cycles for near-Hamiltonian polynomial systems," Journal of Mathematical Analysis and Applications, vol. 348, no. 1, pp. 87-100, 2008.
[13] A. Atabaigi and H. R. Z. Zangeneh, "Bifurcation of limit cycles in small perturbations of a class of hyper-elliptic Hamiltonian systems of degree 5 with a cusp," The Journal of Applied Analysis and Computation, vol. 1, no. 3, pp. 299-313, 2011.

[14] R. Kazemi, H. R. Z. Zangeneh, and A. Atabaigi, "On the number of limit cycles in small perturbations of a class of hyper-elliptic Hamiltonian systems," Nonlinear Analysis: Theory, Methods of Applications, vol. 75, pp. 574-587, 2012.

[15] J. Wang and D. Xiao, "On the number of limit cycles in small perturbations of a class of hyper-elliptic Hamiltonian systems with one nilpotent saddle," Journal of Differential Equations, vol. 250, no. 4, pp. 2227-2243, 2011.

[16] J. Wang, "Estimate of the number of zeros of Abelian integrals for a perturbation of hyperelliptic Hamiltonian system with nilpotent center," Chaos, Solitons \& Fractals, vol. 45, no. 9-10, pp. 1140-1146, 2012.

[17] Y. Xiong, "Bifurcation of limit cycles by perturbing a class of hyper-elliptic Hamiltonian systems of degree five," Journal of Mathematical Analysis and Applications, vol. 411, no. 2, pp. 559$573,2014$. 


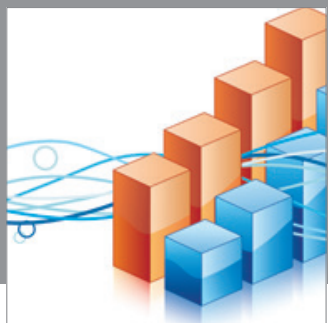

Advances in

Operations Research

mansans

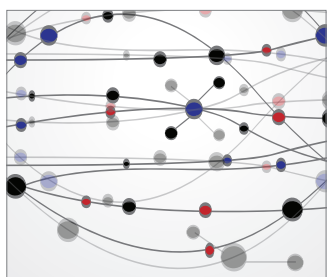

The Scientific World Journal
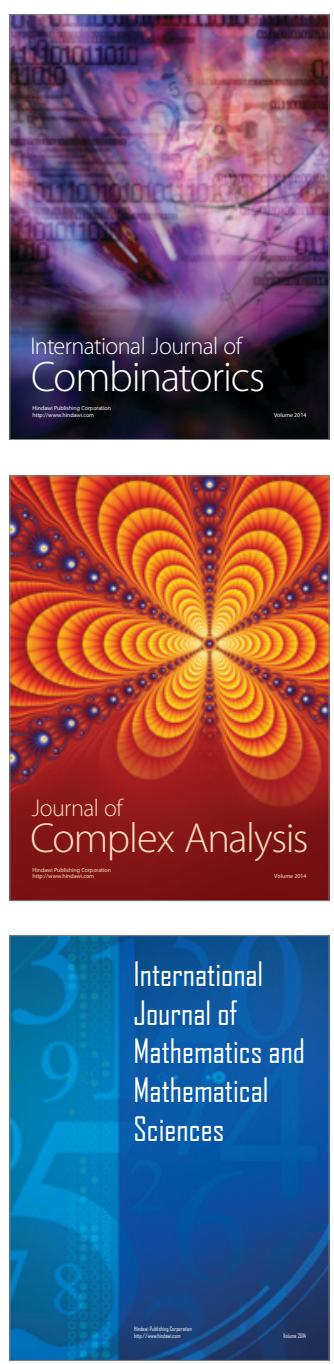
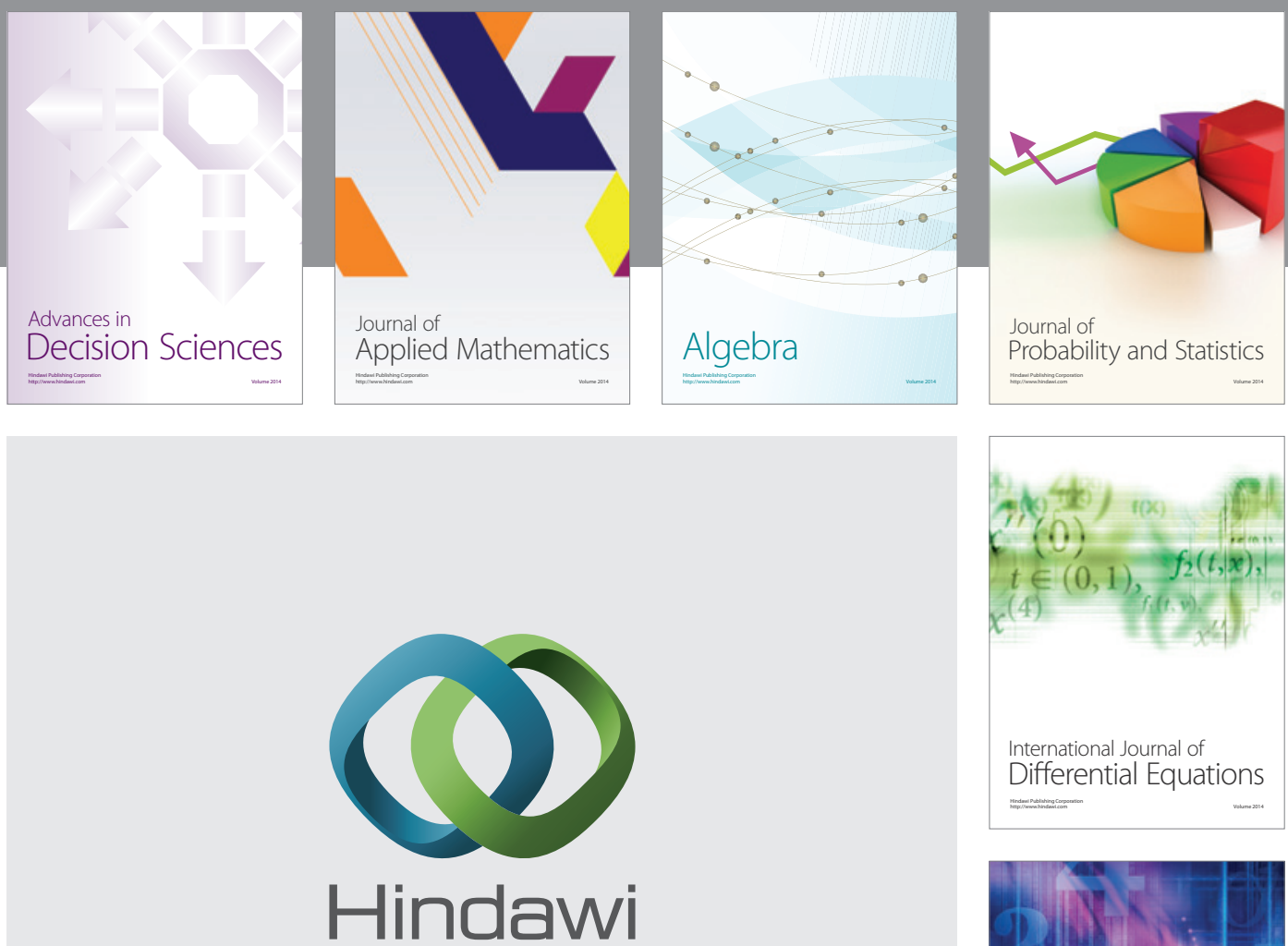

Submit your manuscripts at http://www.hindawi.com
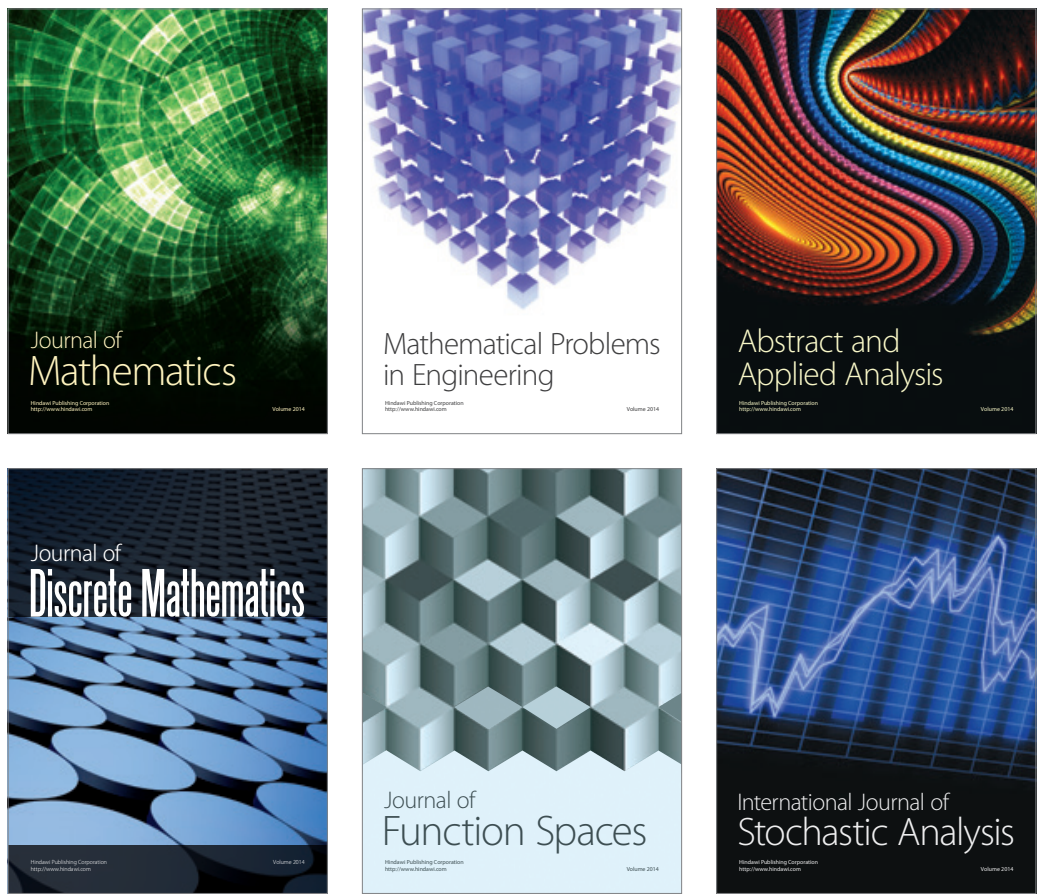

Journal of

Function Spaces

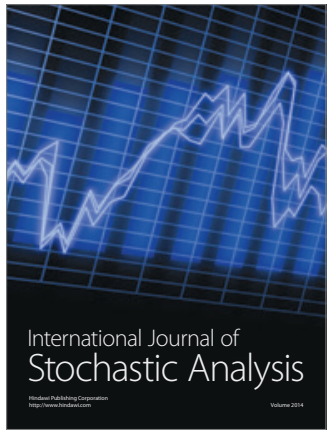

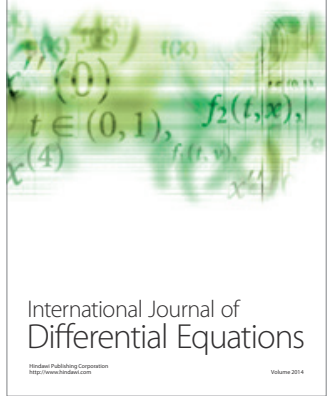
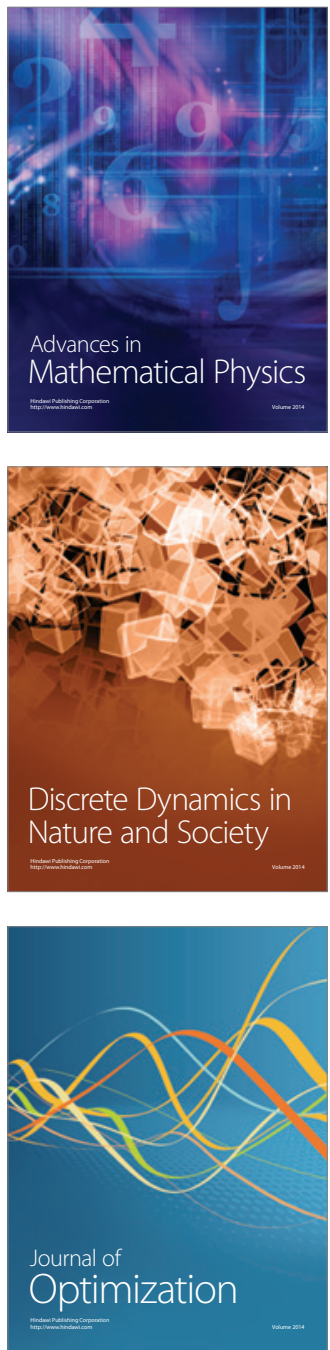